\title{
The impact of a massive star cluster on its surrounding matter in the Antennae overlap region
}

\author{
C. N. Herrera ${ }^{1,2}$ and F. Boulanger ${ }^{3}$ \\ ${ }^{1}$ Institut de Radioastronomie Millimétrique, 300 rue de la Piscine, Domaine Universitaire, 38406 Saint-Martin-d'Hères, France \\ e-mail: herrera@iram.fr \\ 2 National Astronomical Observatory of Japan (NAOJ), 2-21-1 Osawa, Mitaka, 181-8588 Tokyo, Japan \\ 3 Institut d'Astrophysique Spatiale, UMR 8617 CNRS, Université Paris-Sud 11, 91405 Cedex Orsay, France
}

Received 7 March 2016 / Accepted 30 December 2016

\begin{abstract}
Super star clusters (SSCs), likely the progenitors of globular clusters, are one of the most extreme forms of star formation. Understanding how SSCs form is an observational challenge. Theoretical studies establish that, to form such clusters, the dynamical timescale of their parent clouds has to be shorter than the timescale of the disruption of their parent clouds by stellar feedback. However, due to insufficient observational support, it is still unclear how feedback from SSCs acts on the matter surrounding them. Studying feedback in SSCs is essential to understanding how such clusters form. Based on ALMA and VLT observations, we study this process in a SSC in the overlap region of the Antennae galaxies $(22 \mathrm{Mpc})$, a spectacular example of a burst of star formation triggered by the encounter of two galaxies. We analyze a unique massive $\left(\sim 10^{7} M_{\odot}\right)$ and young (1-3.5 Myr) SSC, still associated with compact molecular and ionized gas emission, which suggest that it may still be embedded in its parent molecular cloud. The cluster has two CO velocity components, a low-velocity one spatially associated with the cluster, and a high-velocity one distributed in a bubble-like shape around the cluster. Our results on the low-velocity component suggest that this gas did not participate in the formation of the SSC. We propose that most of the parent cloud has already been blown away, accelerated at the early stages of the SSC evolution by radiation pressure, in a timescale $\sim 1$ Myr. The high-velocity component may trace outflowing molecular gas from the parent cloud. Supporting evidence is found in shock-heated $\mathrm{H}_{2}$ gas and escaping $\mathrm{Br} \gamma$ gas associated with this component. The low-velocity component may be gas that was near the SSC when it formed but not part of its parent cloud or clumps that migrated from the SGMC environment. This gas would be dispersed by stellar winds and supernova explosions. The existing data is inconclusive as to whether or not the cluster is bound and will evolve as a globular cluster. Within $\sim 100 \mathrm{pc}$ of the cluster, we estimate a lower limit for the SFE of $17 \%$, smaller than the theoretical limit of $30 \%$ needed to form a bound cluster. Further higher spatial resolution observations are needed to test and quantify our proposed scenario.
\end{abstract}

Key words. galaxies: individual: Antennae - galaxies: ISM - galaxies: star formation

\section{Introduction}

Super star clusters (SSCs), potentially the progenitors of globular clusters (e.g., Portegies Zwart et al. 2010), are one of the most extreme forms of star formation. They are compact (a few parsec size $)$ and massive $\left(>10^{5} M_{\odot}\right)$ star clusters. SSCs are found in galaxy interactions (e.g., Whitmore \& Schweizer 1995) and starburst dwarf galaxies (e.g., Turner et al. 1998; Beck et al. 2002), as well as in the Milky Way (Clark et al. 2005). The number of such objects greatly increases in galaxy interactions and mergers, common phenomena in the Universe. Therefore, SSCs must be ubiquitous in the Universe.

Understanding the formation and early evolution of SSCs is observationally challenging, thus it is mainly based on theoretical work. Theoretical studies suggest that high external pressures $\left(\sim 10^{7} k_{\mathrm{B}}-10^{8} k_{\mathrm{B}} \mathrm{cm}^{-3} \mathrm{~K}\right)$ are needed to form such compact and massive clusters, conditions typically found in galaxy interactions (Elmegreen \& Efremov 1997; Ashman \& Zepf 2001). The absence of rotational shear in interacting and irregular galaxies may be an additional contribution to SSC formation, which, in spirals, may prevent giant molecular clouds (GMCs) from collapsing into dense clusters (Weidner et al. 2010). High external pressures can accelerate the collapse of molecular clouds, enhancing the star-formation efficiency (SFE, the fraction of gas which turns into stars). If the local ( $\lesssim 1 \mathrm{pc})$ SFE is high ( $\gtrsim 30-50 \%)$, bound cluster formation will occur (Elmegreen \& Efremov 1997; Ashman \& Zepf 2001). Recent hydrodynamical models indicate that in dense star-forming regions, even if the global SFE in a GMC is below 30\%, the local SFE can be higher than 50\%, allowing the formation of bound clusters (Kruijssen et al. 2012). However, observational evidence constraining the SFE in young massive clusters is difficult to obtain. For instance, the SFE of a young ( $\sim 1 \mathrm{Myr}) \mathrm{SSC}$ in the dwarf galaxy NGC 5253 is estimated to be $50 \%$ (Turner et al. 2015) or $\sim 20 \%$ (Smith et al. 2016), depending on the assumed initial mass function.

High local SFE will occur if the local dynamical timescale of the parent molecular cloud (cluster formation timescale) is shorter than the timescale of disruptive processes (i.e., Portegies Zwart et al. 2010). The most invoked disruptive process is the stellar feedback, that is, the interaction of stars with the interstellar medium. The energy and momentum injected by the newly formed stars will unbind and disperse the matter around them. How quickly this occurs is key to the SFE. Studying feedback in SSCs is essential for understanding how such clusters form and evolve. 
The specific role of each stellar feedback mechanism on impeding star formation and driving cloud disruption is a debated topic (see review paper Krumholz et al. 2014). Several stellar feedback mechanisms have been proposed and quantified analytically. Matzner \& McKee (2000) discuss the ability of winds from low-mass stars to drive turbulence and balance dissipation. Matzner (2002) discusses the disruption of clouds by photoionization and expansion of the H II region by the thermal gas pressure. More recent studies have included the impact of the radiation pressure on the expansion of the HII region and the disruption of the parent molecular clouds (Murray et al. 2010; Fall et al. 2010). The mechanical energy associated with supernova explosions and winds from massive stars are also potentially able to disrupt clouds. The mechanical energy would be thermalized in shocks producing high pressure hot plasma, which can drive the expansion of the surrounding matter, provided it remains within the cloud. Observations and theoretical work indicate that this may not be as efficient as originally proposed (Castor et al. 1975), because the plasma is able to flow out through holes in the bubbles very early in the expansion (Harper-Clark \& Murray 2009).

Analytical models and numerical simulations have attempted to explain how parent molecular clouds of massive $\left(\sim 10^{5} M_{\odot}\right)$ star clusters are disrupted (e.g., Murray et al. 2010; Dale \& Bonnell 2011; Dale et al. 2012; Skinner \& Ostriker 2015). Analytical models suggest that radiation pressure is the dominant stellar feedback mechanism close to the clusters where the escape velocity is larger than the sound speed of the H II gas (Krumholz \& Matzner 2009; Murray et al. 2010). But, observational evidence of feedback in individual young $(\lesssim 3 \mathrm{Myr})$ and massive $\left(\gtrsim 10^{5} M_{\odot}\right)$ clusters is still scarce.

SSCs in nearby galaxies are ideal sites to investigate feedback mechanisms of massive clusters. Lopez et al. (2011) have used observations of the 30 Doradus star-forming region in the Large Magellanic Cloud, powered by one of the closest SSCs ( $\gtrsim 5 \times 10^{4} M_{\odot}$, Andersen et al. 2009), to compare the relative values of the pressures associated with radiation, ionized gas, and hot plasma, as a function of position in the nebula. They claim that the embedded massive cluster has broken apart its parent cloud within a time scale shorter than the main sequence life time of their most massive stars, thus before SN explosions become significant. Within the shells close to the core of the central R136 SSC, radiation pressure dominates. In this paper, we carry out a similar study in the Antennae galaxy merger for the most massive and youngest SSC across the overlap region.

The Antennae galaxy (NGC 4038/39) is a nearby (22 Mpc, Schweizer et al. 2008) and well studied merger between two gas-rich spiral galaxies. The region where the two spiral disks interact is known as the overlap region. The molecular gas content is principally constrained to both nuclei and to the overlap region, where it is observed to be fragmented into SuperGiant Molecular Complexes (SGMCs, Wilson et al. 2000). Interferometric observations showed that SGMCs are clumpy and present different velocity components (SMA observations by Ueda et al. 2012, ALMA observations by Herrera et al. 2012 and Whitmore et al. 2014). The Antennae galaxies host a large number of SSCs (Whitmore \& Schweizer 1995; Mengel et al. 2005; Whitmore et al. 2010), which have masses of up to almost $10^{7} M_{\odot}$. We refer to Adamo \& Bastian (2015) for a recent discussion of the fraction of star formation that happens in clusters. In this study we focus on the overlap region, where the most massive $\left(\geq 10^{5} M_{\odot}\right)$, and also the youngest ( $\left.<10 \mathrm{Myr}\right)$ SSCs are located (Mengel et al. 2005), which are spatially associated with SGMCs.
The paper is organized as follows. In Sect. 2, we introduce the datasets used in this paper. In Sect. 3, we present the search in our fields-of-view (FOVs) for SSCs associated with molecular and ionized gas on scales of their parent GMCs. We focus on a unique SSC with both molecular and ionized gas emission, suggesting that it may still be embedded in its parent cloud. This $\mathrm{SSC}, \mathrm{B} 1$, is the most massive SSC in the overlap region. Section 4 presents the physical characteristics of SSC B1 and its parent cloud, and the measurement of their gas emission lines. In Sect. 5, we describe the physical structure of the matter surrounding SSC B1. In Sect. 6, we discuss the role of radiation pressure in disrupting the parent molecular cloud and argue that the bound natal cloud is already disrupted and outflowing gas from this cloud can still be observed. In Sect. 7, we discuss our interpretation of the data. Finally, Sect. 8 gives the conclusions of this paper.

\section{Observations}

Our investigation combines two datasets on the Antennae overlap region. We used observations obtained in February 2011 with the SINFONI imager spectrometer facility on the ESO Very Large Telescope, in the near-IR $K$-band which includes $\mathrm{Br} \gamma$ and several $\mathrm{H}_{2}$ rovibrational lines. The spectral resolving power is $R=4000$ at $\lambda=2.2 \mu \mathrm{m}$. The SINFONI FOV is $8^{\prime \prime} \times 8^{\prime \prime}$ in size. In Herrera et al. (2011) and Herrera et al. (2012), we introduced our four SINFONI pointings across the overlap region, each of them covers a single SGMC. Figure 1 shows the $K$-band emission from the Antennae overlap region, where we mark our four SINFONI FOVs with dashed boxes. In this paper, we use the SINFONI observations of SGMC 4/5. The angular resolution is 0, ! $7 \times 0$ '.6 (full width at half maximum, FWHM). We computed the velocity resolution at different wavelengths by fitting Gaussian curves to the sky-lines (see Table 3 ). Details of the data reduction can be found in Herrera et al. (2012).

We combined the previous dataset with archive observations done with the ALMA interferometer in the Cycle 0 early science process between July and November, 2012, which covered the entire Antennae overlap region (PI: B. Whitmore, Whitmore et al. 2014). The integration time on source was $3 \mathrm{~h}$ and the number of antennas varied between 14 and 24. These observations, done in Band 7 (345 GHz), include the $\mathrm{CO}(3-2)$ line emission as well as dust continuum emission. The data was retrieved from the ALMA science archive fully reduced by the ALMA team. We imaged the data using the CLEAN algorithm in CASA, by defining cleaning boxes enclosing the gas emission at each channel. The native spectral resolution of the observations corresponds to a channel width of $\sim 0.5 \mathrm{MHz}\left(\sim 0.5 \mathrm{~km} \mathrm{~s}^{-1}\right)$. To increase the signal-to-noise ratio, we smoothed the channels to a velocity resolution of $10 \mathrm{~km} \mathrm{~s}^{-1}$. The synthesized beam FWHM is $0^{\prime \prime} .55 \times 00^{\prime \prime} .43$, which corresponds to a lineal scale of $59 \mathrm{pc} \times 46 \mathrm{pc}$ at the distance of the Antennae. We finally corrected the image by the primary beam response. We also used archive $\mathrm{CO}(2-1)(230 \mathrm{GHz})$ observations obtained with ALMA in the Band 6 during the science verification process. These observations were presented by Espada et al. (2012). Data reduction was performed by the ALMA team. We corrected the data cube by the primary beam response. The synthesized beam is 0 '. $86 \times 1^{\prime \prime} .67\left(\mathrm{PA}=77^{\circ}\right)$, and the velocity resolution is $20 \mathrm{~km} \mathrm{~s}^{-1}$.

\section{Search for embedded clusters}

In this section, we show how we selected a unique SSC across the Antennae overlap region to study the disruption of its parent 
Table 1. SSCs within the area observed with SINFONI.

\begin{tabular}{cccccc}
\hline \hline $\begin{array}{c}\text { SSC } \\
\text { ID }\end{array}$ & $\begin{array}{c}\text { Age }^{a} \\
\text { Myr }\end{array}$ & $\begin{array}{c}\text { Mass } \\
10^{6} M_{\odot}\end{array}$ & Ref. $^{b}$ & $\begin{array}{c}\text { Flux H}_{2} \\
\text { erg s}^{-1}\end{array}$ & $\begin{array}{c}\text { Flux Br } \\
\mathrm{cm}^{-2}\end{array}$ \\
\hline $\mathrm{D}$ & 3.9 & 1.4 & {$[1]$} & $<2.9 \times 10^{-16}$ & $2.1 \pm 0.1 \times 10^{-15}$ \\
30 & 1.45 & 0.32 & {$[2]$} & & \\
\hline $\mathrm{D} 1$ & 6.1 & 1.6 & {$[1]$} & $<1.1 \times 10^{-16}$ & $<1.5 \times 10^{-16}$ \\
3 & - & - & {$[2]$} & & \\
\hline $\mathrm{D} 2$ & 5.4 & 0.8 & {$[1]$} & $<1.1 \times 10^{-16}$ & $6.7 \pm 0.3 \times 10^{-16}$ \\
\hline $\mathrm{C}$ & 5.7 & 4.1 & {$[1]$} & $<1.0 \times 10^{-16}$ & $<9.9 \times 10^{-17}$ \\
28 & 4.8 & 1.2 & {$[2]$} & & \\
\hline B1 $^{c}$ & 3.5 & 4.2 & {$[1]$} & $2.9 \pm 0.2 \times 10^{-15}$ & $2.2 \pm 0.1 \times 10^{-14}$ \\
16 & 1 & 6.8 & {$[2]$} & & \\
\hline
\end{tabular}

Notes. Fluxes are not corrected for extinction. ${ }^{(a)}$ Errors in ages estimated by Gilbert \& Graham (2007) are derived from Starburst99 fits to the Bry EW and are typically below 0.1 Myr. Cluster D2 has a larger error on the $\mathrm{Br} \gamma \mathrm{EW}$, the error on the age is $5.4_{-1.4}^{+0.4}$. (b) [1] Gilbert \& Graham (2007), [2] Table 8 in Whitmore et al. (2010). Masses estimated by Gilbert \& Graham (2007) are not corrected by extinction. ${ }^{(c)}$ SSC first identified by Whitmore \& Schweizer (1995) as WS80.

cloud. We first introduce the SSCs population over the overlap region and then we explain how we used the SINFONI data, which cover four FOVs in the overlap region, to search for compact line emission associated with SSCs.

All clusters younger than $\sim 10$ Myr have significant ionizing flux. For SSCs embedded in their parent cloud, it is expected to detect $\mathrm{Br} \gamma$ line emission from its $\mathrm{H}$ II region, which should be compact $(\sim 100 \mathrm{pc})$ and barely resolved by the arcsecond spatial resolution of near-IR ground-based observations. We also expect to detect $\mathrm{H}_{2}$ line emission from photo dissociated regions (PDRs; e.g., Habart et al. 2011). For older SSCs, the $\mathrm{Br} \gamma$ emission would be more extended because the ionizing photons travel into the SGMCs and the more diffuse ISM, over distances larger than the size of their parent cloud. The detection of line emission associated with SSCs in the SINFONI data-cube of the Antennae overlap region is not straightforward because the SGMCs, where SSCs are found, show complex velocity structures and some of them are associated with several SSCs (Herrera et al. 2012). We face two main difficulties. The first one is to isolate the emission associated with the SSC from that of SGMCs, and the second one is to isolate different sources within SGMCs. We focus on SSCs, which are isolated. We selected a few SSCs previously studied by Gilbert \& Graham (2007), based on single-slit spectroscopic Br $\gamma$ observations. Gilbert \& Graham (2007) estimated ages and masses from the observed $\mathrm{Br} \gamma$ equivalent width and the cluster stellar magnitude, employing the stellar population synthesis model Starburst $99^{1}$ and adopting a Kroupa initial mass function (IMF). These SSCs are young ( $\lesssim 6 \mathrm{Myr}$ ) and massive $\left(>8 \times 10^{5} M_{\odot}\right)$, and lie within the four SINFONI fields presented in Herrera et al. (2012). These are the clusters D, D1, and D2 in SGMC 1, C in SGMC 2 and B1 in SGMC 4/5, which are highlighted in Fig. 1 and listed in Table 1. We also list in Table 1 the properties of these clusters estimated by Whitmore et al. (2010) using integrated photometry in the filters $U B V I H_{\alpha}$ complemented with population synthesis models. For each of these SSCs, we searched in the SINFONI data for compact (diameter $\sim 1^{\prime \prime}$ ) gas emission associated with the SSCs, to identify which ones are still embedded in their parent clouds.

\footnotetext{
1 http://www.stsci.edu/science/starburst99/docs/ default.htm
}

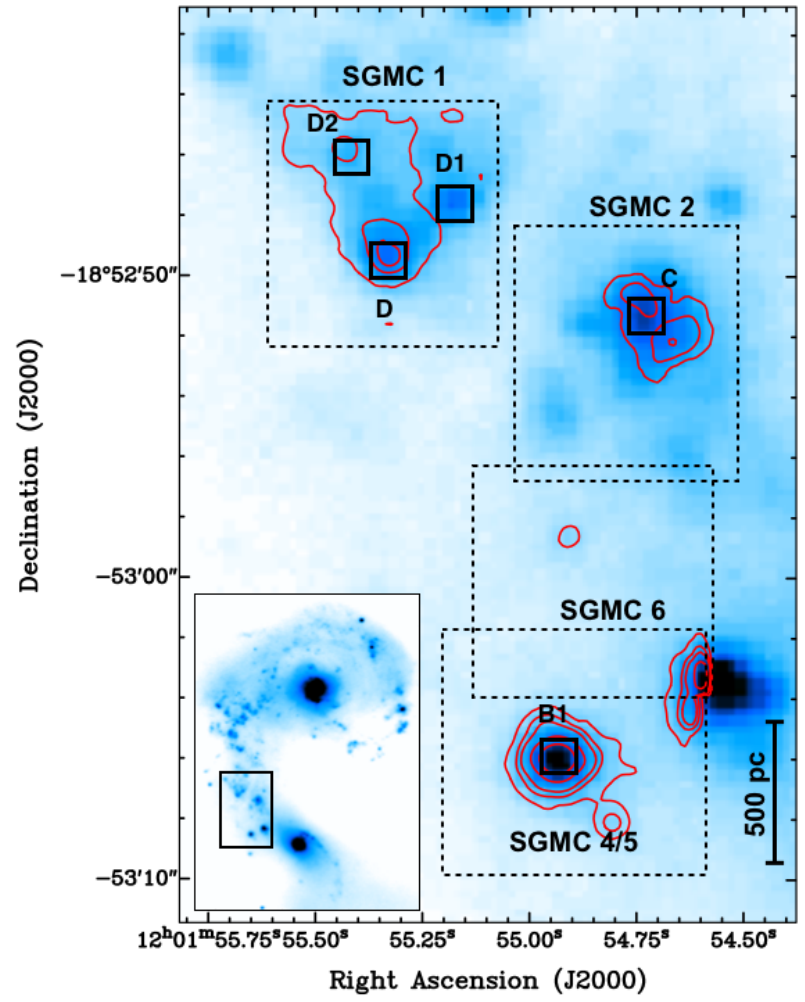

Fig. 1. $K$-band emission from the overlap region obtained with the CFHT (Herrera et al. 2011) overlaid with Br $\gamma$ contours from the SINFONI observations. Small black squares highlight the position of the SSCs lying within the four SINFONI FOVs, which are marked with dashed boxes. An inserted image of the Antennae galaxies shows, in a black rectangle, the relative position of the overlap region in the merger.

For each SSC, we obtained the $K$-band spectrum by circular aperture photometry. The aperture diameter is $\sim 1^{\prime \prime} \cdot 3(140 \mathrm{pc})$, twice the SINFONI seeing size. We subtracted the nearby background measured within an annulus of 0 '.65 width, the seeing of our SINFONI data. Within the SINFONI spectra, we measured the molecular and ionized emission line fluxes, listed in Table 1. Upper limits for the fluxes were estimated as the standard deviation of aperture photometry of several positions in the integrated line image. In three of the selected SSCs - D, D2 and B1 - we detect $\mathrm{Br} \gamma$. These clusters have ages $\lesssim 5 \mathrm{Myr}$ and masses $\gtrsim 10^{6} M_{\odot}$. The young and massive SSC C in SGMC 2 , has an ionized nebula larger than the aperture size used to search for compact emission (Herrera et al. 2011). Other clusters may not be detected in the line emission due to the difficulty of isolating their emission from the background. This is a problem for the less massive clusters. Gilbert \& Graham (2007) detected Bry emission for all clusters using an aperture size of $2^{\prime \prime}(\sim 220 \mathrm{pc})$, which includes not only compact $\mathrm{H}$ II regions but also extended emission. In only one cluster, SSC B1, we do detect both $\mathrm{H}_{2}$ and $\mathrm{Br} \gamma$ compact emission, suggesting that this cluster may still be embedded in its parent molecular cloud.

Figures 2 and 3 show the ratio between the line fluxes (both $\mathrm{H}_{2}$ 1-0 $\mathrm{S}(1)$ and $\mathrm{Br} \gamma$ ) and cluster masses versus cluster ages for six and five sources, respectively. The figures include all of the SSCs extracted from Gilbert \& Graham (2007), Fig. 2 also includes the molecular compact source PCC 1 presented in Herrera et al. (2011). The $\mathrm{H}_{2}$-to-stellar mass and $\mathrm{Br} \gamma$-to-stellar mass ratio are normalized to the value for B1. Figure 2 shows that the $\mathrm{H}_{2}$ emission per unit of stellar mass decreases sharply, by more than one order of magnitude for ages of approximately 


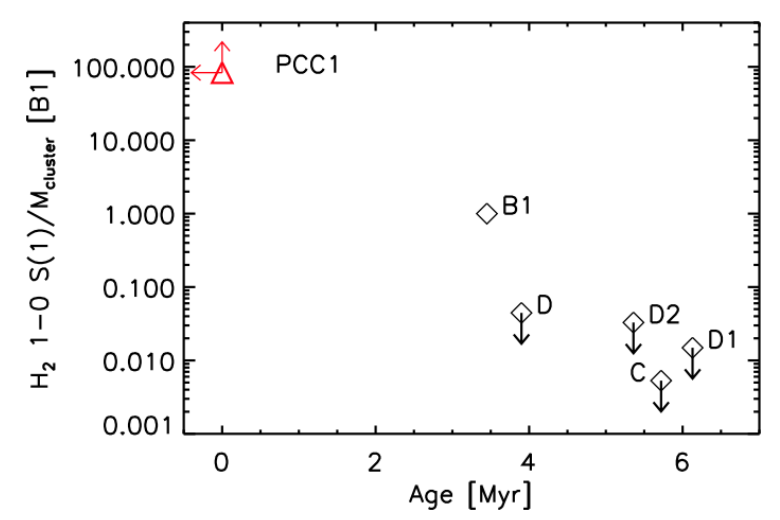

Fig. 2. $\mathrm{H}_{2}$ 1-0 S(1) emission over the star-cluster mass, for SSCs with different ages and PCC 1 (Herrera et al. 2011), observed in the SINFONI fields. Since both the masses and $\mathrm{H}_{2}$ 1-0 S(1) fluxes depend on the extinction in the same way, the $\mathrm{H}_{2}$-to-stellar mass ratio is independent of extinction. PCC 1 has a lower limit since it has not yet formed massive stars. The $\mathrm{H}_{2}$-to-stellar mass ratio is normalized to the value for B1.

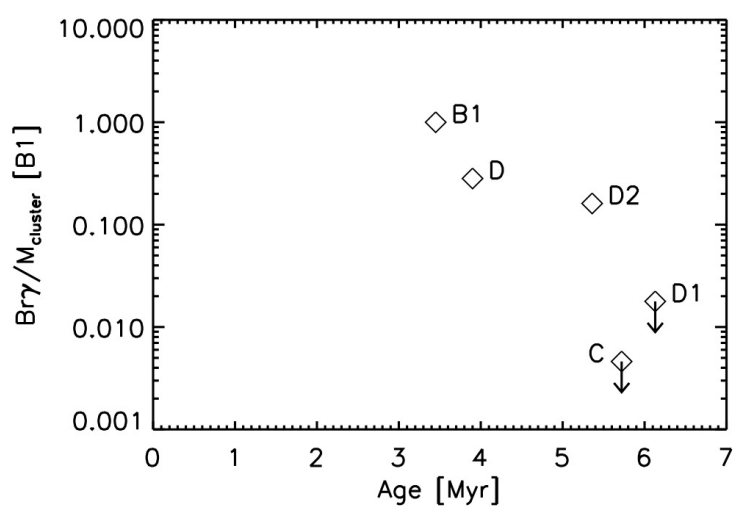

Fig. 3. Br $\gamma$ emission over the star cluster mass, for the SSCs in the SINFONI fields. As for $\mathrm{H}_{2}$ (see Fig. 2), the $\mathrm{Br} \gamma$-to-stellar mass ratio is extinction independent. The ratio is normalized to the value for B1.

3.5 Myr. Only PCC 1 and B1 show clear $\mathrm{H}_{2}$ emission. Sources without an obvious $\mathrm{H}_{2}$ detection are older than B1. This suggests that the disruption of the parent clouds by the newly formed stars occurs within a few Myr ( $<5 \mathrm{Myr})$. Figure 3 shows a large amount of scatter. The $\mathrm{Br} \gamma$ emission does not decrease as fast as the $\mathrm{H}_{2}$ emission. The comparison between D and D1 in SGMC 1 gives us an idea of the time-scale for the disruption of $\mathrm{H}$ II regions in the overlap region. Both clusters have similar masses $\left(\sim 10^{6} M_{\odot}\right)$ but only $\mathrm{D}$, which is $2 \mathrm{Myr}$ younger, is associated with compact ionized gas emission. SSC D1, $\sim 6 \mathrm{Myr}$ old and with a mass of a few times $10^{5} M_{\odot}$, has already cleared out all or most of its parent cloud. Our findings agree with the upper limit for the parent cloud disruption timescale of $<5 \mathrm{Myr}$ estimated for SSCs in a sample of nearby galaxies and other regions of the Antennae pair (Bastian et al. 2014).

In the rest of the paper, we focus on SSC B1 (also identified as "WS80" in other references), the only SSC across the overlap region associated with bright compact ionized and molecular gas emission. This cluster coincides with a bright emission peak in the mid-IR discovered by ISO, accounting for $15 \%$ of the midIR flux from the whole Antennae (Mirabel et al. 1998). SSC B1 is the brightest compact radio source at 4 and $6 \mathrm{~cm}$ in the overlap region (Neff \& Ulvestad 2000). It is also one of the brightest sources in the $K$-band and $\mathrm{Br} \gamma$ emission, in the near-IR observations presented by Gilbert et al. (2000) and our SINFONI data.
Gilbert et al. (2000) infers that the nebular part of this cluster is a compact, dense H II region $\left(n=10^{4} \mathrm{~cm}^{-3}\right)$. Whitmore \& Zhang (2002) find that SSC B1 is the intrinsically brightest cluster in the Antennae merger. The ALMA maps published by Herrera et al. (2012, see their Fig. 1) and by Whitmore et al. (2014, their Fig. 10) show that it coincides with a CO emission peak. SSC $\mathrm{B} 1$ is the more massive and youngest stellar cluster within our SINFONI fields (Table 1), which are the two fundamental properties to study embedded SSCs without confusion.

\section{Physical properties of the SSC and GMC}

SSC B1 in SGMC 4/5 is associated with compact ionized and molecular gas emission. This suggests that B1 may still be embedded in its parent molecular cloud. It is the target that we use to study how massive SSCs disperse their parent molecular clouds. In this section, we use the SINFONI and ALMA observations to characterize the physical properties of the cluster and its parent cloud.

\subsection{SSC B1}

We quantify the number of ionizing photons $N_{\text {Lyc }}$ from the cluster. The radio flux of SSC B1 at 4 and $6 \mathrm{~cm}$ has a thermal spectral index (Neff \& Ulvestad 2000). Both thermal radio emission and hydrogen recombination lines originate within ionized regions and are proportional to $N_{\text {Lyc }}$, but the radio emission, unlike the $\mathrm{Br} \gamma$ emission, is not affected by dust extinction. From the thermal radio emission, we estimate $N_{\text {Lyc }}$ by using Eq. (1) in Roman-Lopes \& Abraham (2004). The radio fluxes at 4 and $6 \mathrm{~cm}$ are listed in Tables 4 and 3 in Neff \& Ulvestad (2000, brightest source in their region \#2), respectively, which gives a mean value of $N_{\mathrm{Lyc}}=2.2 \pm 0.1 \times 10^{53}$ phot s ${ }^{-1}$. Gilbert \& Graham (2007) report a value $\sim 1.4$ lower, estimated from the extinction corrected $\mathrm{Br} \gamma$ flux. Uncertainties in the estimation of $N_{\text {Lyc }}$ come from the unknown fraction of ionizing photons that do not escape the H II region but are absorbed by dust. Such a fraction is difficult to quantify. Observations of Galactic H II regions yield a photoionizing fraction between 0.3 and 1.0 (see Fig. 10 in Draine 2011).

The age and stellar mass of SSC B1 are hard to constrain and discrepancies are found when using different methods. From the $K$-band spectrum of SSC B1 (Fig. 5), we can set an upper limit to the age of $\sim 6 \mathrm{Myr}$ due to the absence of $\mathrm{CO}$ bandheads at $2.3 \mu \mathrm{m}$. This age limit agrees with the ages estimated by Whitmore \& Zhang (2002) and Whitmore et al. (2010) of 2 Myr and $1 \mathrm{Myr}$, respectively, comparing UBVIH $\alpha$ photometry with population synthesis models. It also agrees with the cluster age derived by Gilbert \& Graham (2007) of 3.5 Myr from the equivalent width $(\mathrm{EW})$ of the $\mathrm{Br} \gamma$ line of $255 \pm 8 \AA$, and our own estimation of the Br $\gamma$ EW from our SINFONI observations of $295 \pm 24 \AA(3.5 \pm 0.7 \mathrm{Myr})$. The error bar in our estimation of the age is a statistical value from the EW measurement. Systematical uncertainties from the unknown fraction of ionizing photons escaping the $\mathrm{H}$ II region are larger. The stellar mass is estimated to be $6.6 \times 10^{6}$ and $6.8 \times 10^{6} M_{\odot}$ by Whitmore \& Zhang (2002) and Whitmore et al. (2010), respectively.

We compute the cluster bolometric luminosity using the Starburst99 models, for a given age and mass of the cluster. Using the age and mass estimated by Whitmore et al. (2010), we find $L_{\mathrm{cl}}=5.3 \times 10^{9} L_{\odot}$. Note that the bolometric luminosity has a nonlinear dependence with age, and the highest value is observed between 2 and 4 Myr. For 3.5 Myr, the age estimated by the $\mathrm{Br} \gamma$ 
Table 2. Estimated properties of B 1 cluster in SGMC 4/5 and its parent molecular cloud.

\begin{tabular}{|c|c|}
\hline$N_{\text {Lyc }}$ & $2.2 \pm 0.1 \times 10^{53}$ phot s $^{-1}$ \\
\hline Stellar age ${ }^{a}$ & $1-3.5 \mathrm{Myr}$ \\
\hline Stellar mass & $6.8 \times 10^{6} M_{\odot}$ \\
\hline$L_{\mathrm{cl}}$ & $5.3 \times 10^{9} L_{\odot}$ \\
\hline$A_{\mathrm{K}}$ & $0.8 \mathrm{mag}$ \\
\hline $\mathrm{Br} \gamma$ emission size & $70 \mathrm{pc}$ \\
\hline $\mathrm{Br} \gamma$ equivalent width & $295 \pm 28 \AA$ \\
\hline$K$-band emission size & $66 \mathrm{pc}$ \\
\hline $\mathrm{H}_{2}$ emission size & $<210 \mathrm{pc}$ \\
\hline GMC size $^{b}$ & $104 \mathrm{pc}$ \\
\hline GMC gas mass $M_{\mathrm{H}}$ & $3.1 \pm 0.1 \times 10^{7} M_{\odot}$ \\
\hline Mean gas density ${ }^{c} n_{\mathrm{H}}$ & $2.1 \pm 0.1 \times 10^{3} \mathrm{~cm}^{-3}$ \\
\hline$N_{\mathrm{H}}^{c, d}$ & $4.5 \pm 0.3 \times 10^{23} \mathrm{H} \mathrm{cm}^{-2}$ \\
\hline
\end{tabular}

Notes. ${ }^{(a)}$ The listed values are those from Whitmore et al. (2010) and that fitted from Br $\gamma$ EW (see text). ${ }^{(b)}$ Size (FWHM) measured from continuum emission at $345 \mathrm{GHz} .{ }^{(c)}$ Error bar only includes the error on the cloud mass. ${ }^{(d)}$ Column density at the center of the GMC, computed as $N_{\mathrm{H}}=M_{\mathrm{H}} /$ Area.

EW, the luminosity is $\sim 40 \%$ higher. The mass, age, $N_{\mathrm{Lyc}}$, and $L_{\mathrm{cl}}$ values of the B1 cluster are listed in Table 2.

In this paper, we choose to use the mass estimated by Whitmore et al. (2010), which is obtained at high angular resolution (HST observations), and thus better resolving the stellar emission out of the nebular emission. Additionally, unlike our estimation of the stellar mass from the $N_{\text {Lyc }}$ value, it does not have uncertainties on the photoionization fraction that escapes the H II region.

\subsection{Spatial extent of the ionized and molecular gas associated with SSC B1}

We used the $\operatorname{Br} \gamma$ line to measure the extent of the ionized gas and the continuum emission at $345 \mathrm{GHz}$ for that of the molecular gas. This emission is optically thin and, unlike the near-IR $\mathrm{H}_{2}$ line emission, is a tracer of the column density. The size of the ionized emission associated with the cluster was measured by fitting Gaussian curves to the $\mathrm{Br} \gamma$ emission profiles. The observed size is $0 \prime \prime 85 \times 1{ }^{\prime \prime} .02$ (FWHM), which, after correction by the seeing, gives a geometric mean of 0 '!67 ( FWHM $=70 \mathrm{pc}$, listed in Table 2). A 2D Gauss fit of the $K$-band continuum emission gives a similar observed size of $00^{\prime \prime} 79 \times 1^{\prime \prime}$.02 (FWHM), yielding a seeing-corrected geometric mean of $00^{\prime \prime} 62$ (66 pc).

The size of the molecular cloud was measured by fitting a 2D Gaussian function to the continuum emission. We first subtracted the emission seen to be associated with a water maser detected by Brogan et al. (2010) at the position $\alpha: 12^{\mathrm{h}} 01^{\mathrm{m}} 54.9959$, $\delta$ : $-18^{\circ} 53^{\prime} 05^{\prime \prime}$.543, $\sim 0^{\prime \prime}$.7 away from SSC B1 (see Fig. 6, peak emission in the continuum at $345 \mathrm{GHz}$ ). The absence of a $\mathrm{Br} \gamma$, $K$-band continuum or $\mathrm{H}_{2}$ counterpart suggests that the water maser is an extremely young star forming region. We measured the size (FWHM) of the emission associated with the water maser to be 0 .'76 ( $~ 80 \mathrm{pc})$. We created a 2D Gaussian array, centered at the position of the maser, with a peak emission of that seen in the continuum emission at the position of the maser. A multiplicative factor of 0.5 is found to minimise the dispersion of the residuals after subtracting the water maser contribution and the 2D Gaussian fit of the GMC emission. Figure 4 displays the result of the water maser subtraction, that is, the continuum emission associated with SSC B1. The black dashed

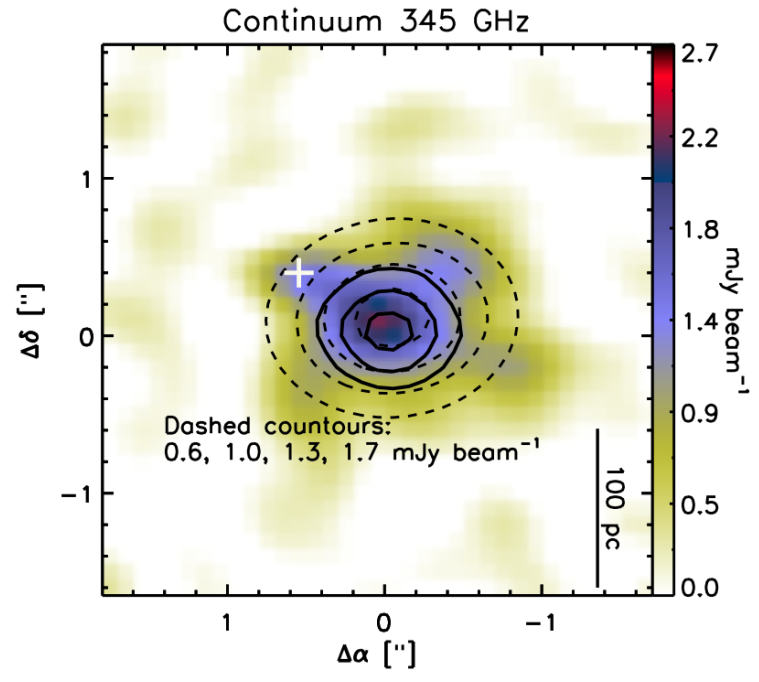

Fig. 4. Continuum emission at $345 \mathrm{GHz}$. Emission from $\mathrm{H}_{2} \mathrm{O}$ maser was subtracted. The white cross marks the position of the $\mathrm{H}_{2} \mathrm{O}$ maser. Solid black lines are the $50 \%, 70 \%$, and $90 \%$ of the peak of the $\mathrm{Br} \gamma$ line emission (see Fig. 6). Dashed black contours correspond to the 2D Gaussian fit to the continuum emission. Offset positions are relative to $\alpha: 12^{\mathrm{h}} 01^{\mathrm{m}} 54 \mathrm{~s} 95, \delta:-18^{\circ} 53^{\prime} 05^{\prime \prime} 6 \mathrm{~J} 2000.0$. The offset position $(0,0)$ corresponds to the position of the SSC B1, which coincides with the continuum peak emission.

contours correspond to the 2D Gaussian fit to this emission, black solid contours represent the Bry emission, and the white cross shows the position of the water maser. The peak of the continuum emission coincides with the position of the SSC B1, offset $(0,0)$. The observed source size (FWHM) is 0 '!96 $\times 11^{\prime \prime} \cdot 22$, which gives a geometrical mean, after beam correction, of 0.98 $(F W H M=104 \mathrm{pc})$. This is the spatial extent of the molecular cloud seen to be associated with SSC B1. It is larger than the $\mathrm{Br} \gamma$ extent, which suggests that the ionizing emission comes from within the cloud. Gaussian fits to the $\mathrm{H}_{2} 1-0 \mathrm{~S}(1)$ emission profile yield a size of $2^{\prime \prime} .0 \times 22^{\prime \prime} 1$ (FWHM), which, after correction by the seeing, gives a geometric mean of $2^{\prime \prime}(F W H M=210 \mathrm{pc}$, listed in Table 2). This value is larger than the GMC size. This indicates that part of the $\mathrm{H}_{2}$ emission comes from the SGMC and is not directly associated with SSC B1.

\subsection{Intensities of the ionized and molecular gas associated with SSC B1}

In this section, we describe the measurements of the $\mathrm{CO}$ and near-IR line fluxes $\left(\mathrm{H}_{2}, \mathrm{Br} \gamma, \mathrm{He} \mathrm{I}\right.$, and $\left.\mathrm{Br} \delta\right)$, listed in Table 3.

The $K$-band spectrum of SSC B1 is presented in Fig. 5. Several ionized and molecular lines are detected; we highlight the main ones. This spectrum is obtained by integrating the flux within an aperture of twice the FWHM observed in Br $\gamma$ (1".9 diameter), and subtracting a background estimated within an annulus of 0".65 width (the size of the seeing disk of the SINFONI data). The same figure shows a zoom on the Br $\gamma$ and $\mathrm{H}_{2}$ 1-0 S(1) lines. Br $\gamma$ is one order of magnitude brighter than the $\mathrm{H}_{2}$ 1-0 $\mathrm{S}(1)$ line. Since the $\mathrm{H}_{2}$ spatial extent is larger than that of $\mathrm{Br} \gamma$, the $\mathrm{H}_{2}$ fluxes measured from this $K$-band spectrum represent an approximate estimate of the $\mathrm{H}_{2}$ emission associated with SSC B1. For a larger aperture of 4".2 diameter, twice the FWHM of the $\mathrm{H}_{2}$ emission profile, with no background subtraction, since it is negligible, we measured a $\mathrm{H}_{2} 1-0 \mathrm{~S}$ (1) flux four times larger. The aperture size of $4 " .2$ is likely to be dominated by the SGMC (conservative upper limit). Measurements of the 


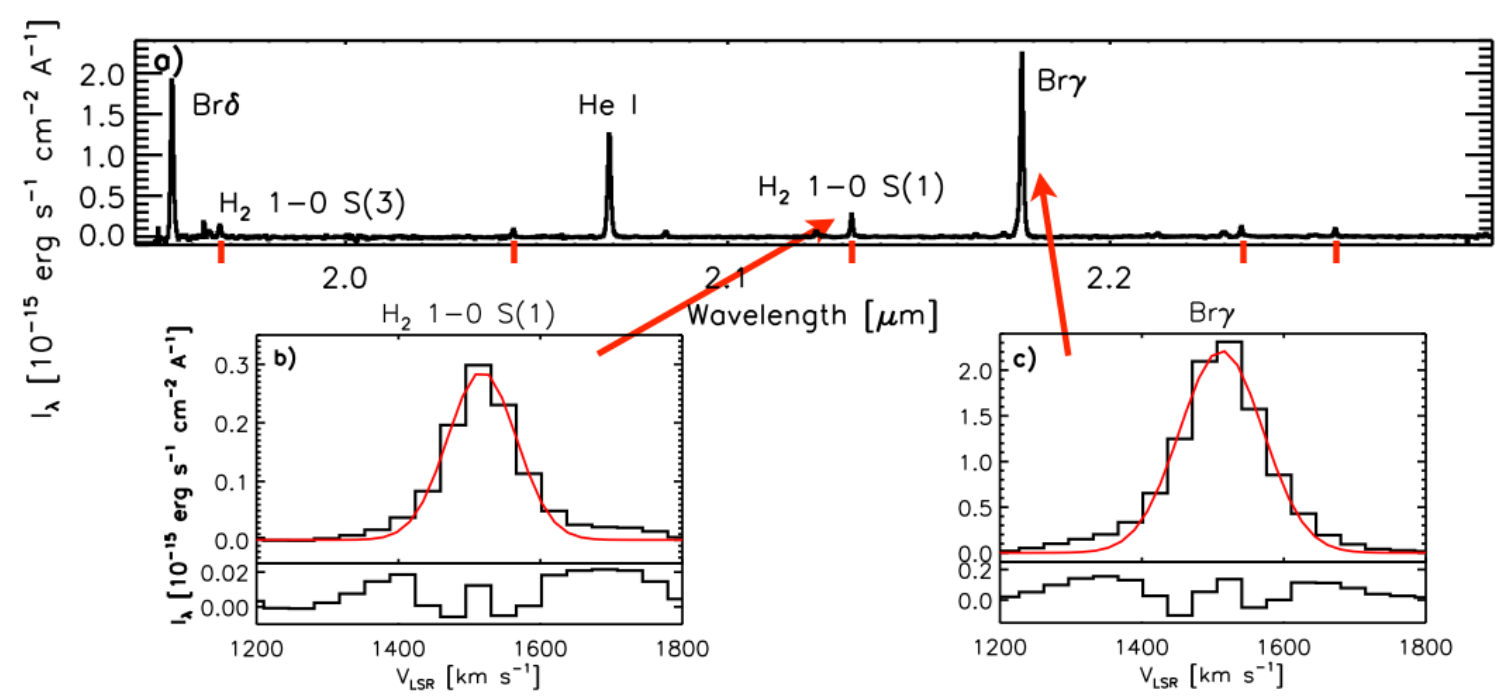

Fig. 5. Upper panel: $K$-band spectrum of SSC B1, obtained from aperture photometry based on the $\mathrm{Br} \gamma$ line. Ionized gas lines are the brightest lines across the spectrum. We mark the position of the $\mathrm{H}_{2}$ rovibrational lines with short red lines. Lower panels: zoom in the $\mathrm{H}_{2} 1-0 \mathrm{~S}(1)$ and $\mathrm{Br} \gamma$ lines. Red lines represent the fitted Gaussian curves to each line, and residuals are plotted in the bottom part. Table 3 lists the parameters of the Gaussian fits.

Table 3. Line parameters measured from SSC B1 in SGMC 4/5.

\begin{tabular}{ccccccc}
\hline \hline Line & $\begin{array}{c}\text { Rest wavelength } \\
\mu \mathrm{m}\end{array}$ & $\begin{array}{c}\text { Flux } \\
\mathrm{erg} \mathrm{s}^{-1} \mathrm{~cm}^{-2}\end{array}$ & $\begin{array}{c}V_{\mathrm{LSR}} \\
\mathrm{km} \mathrm{s}^{-1}\end{array}$ & $\begin{array}{c}F W H M^{b} \\
\mathrm{~km} \mathrm{~s}^{-1}\end{array}$ & $\begin{array}{c}\text { Resolution } \\
\mathrm{km} \mathrm{s}^{-1}\end{array}$ & $\begin{array}{c}F W H M^{c} \\
\mathrm{~km} \mathrm{~s}^{-1}\end{array}$ \\
\hline $\mathrm{Br} \delta^{d}$ & 1.94509 & $1.8 \pm 0.1 \times 10^{-14}$ & $1499 \pm 3$ & $147 \pm 5$ & $129 \pm 6$ & $67 \pm 10$ \\
$\mathrm{HeI}$ & 2.05869 & $1.4 \pm 0.1 \times 10^{-14}$ & $1521 \pm 3$ & $143 \pm 7$ & $106 \pm 3$ & $96 \pm 10$ \\
$\mathrm{HeI}$ & 2.1127 & $6.5 \pm 0.5 \times 10^{-16}$ & $1511 \pm 3$ & $126 \pm 5$ & $101 \pm 6$ & $76 \pm 11$ \\
$\mathrm{Br} \gamma$ & 2.16612 & $2.4 \pm 0.2 \times 10^{-14}$ & $1512 \pm 3$ & $137 \pm 7$ & $87 \pm 4$ & $106 \pm 9$ \\
$\mathrm{H}_{2} 1-0 \mathrm{~S}(3)$ & 1.95756 & $1.7 \pm 0.1 \times 10^{-15}$ & $1501 \pm 4$ & $144 \pm 4$ & $129 \pm 6$ & $64 \pm 15$ \\
$\mathrm{H}_{2} 1-0 \mathrm{~S}(2)$ & 2.03376 & $9.8 \pm 0.7 \times 10^{-16}$ & $1518 \pm 3$ & $129 \pm 5$ & $106 \pm 3$ & $73 \pm 10$ \\
$\mathrm{H}_{2} 1-0 \mathrm{~S}(1)$ & 2.12183 & $2.5 \pm 0.2 \times 10^{-15}$ & $1518 \pm 3$ & $113 \pm 6$ & $101 \pm 6$ & $51 \pm 17$ \\
$\mathrm{H}_{2} 1-0 \mathrm{~S}(0)$ & 2.22329 & $1.3 \pm 0.1 \times 10^{-15}$ & $1511 \pm 3$ & $118 \pm 4$ & $86 \pm 6$ & $80 \pm 9$ \\
$\mathrm{H}_{2} 2-1 \mathrm{~S}(1)$ & 2.24772 & $9.6 \pm 0.8 \times 10^{-16}$ & $1515 \pm 3$ & $109 \pm 6$ & $86 \pm 6$ & $67 \pm 12$ \\
\hline & $\mathrm{GHz}$ & $\mathrm{Jy} \mathrm{km} \mathrm{s}$ & $\mathrm{km} \mathrm{s}^{-1}$ & $\mathrm{~km} \mathrm{~s}^{-1}$ & $\mathrm{~km} \mathrm{~s}^{-1}$ & $\mathrm{~km} \mathrm{~s}^{-1}$ \\
\hline $\mathrm{CO}(2-1)^{e}$ & 230.538 & $110 \pm 2$ & $1504.4 \pm 0.2$ & $60.5 \pm 0.4$ & 20 & $57.1 \pm 0.2$ \\
& & $80 \pm 2$ & $1612 \pm 1$ & $106 \pm 2$ & 20 & $104 \pm 2$ \\
$\mathrm{CO}(3-2)^{f}$ & 345.796 & $153 \pm 4$ & $1502 \pm 1$ & $59 \pm 1$ & 10 & $58 \pm 1$ \\
$\mathrm{CO}(3-2)^{g}$ & & $115 \pm 6$ & $1594 \pm 2$ & $100 \pm 5$ & 10 & $100 \pm 5$ \\
\hline
\end{tabular}

Notes. The error in the fluxes for all the lines includes the $10 \%$ of absolute flux uncertainty and rms per channel. Errors in the central velocities include the uncertainty on the $\mathrm{OH}$ line identification $\left(\sim 1.5-3 \mathrm{~km} \mathrm{~s}^{-1}\right)$. ${ }^{(a)}$ Near-IR fluxes are not corrected for extinction. ${ }^{(b)}$ Observed line-widths without correction for the spectral resolution. ${ }^{(c)}$ Line-widths corrected by the spectral resolution. ${ }^{(d)} \mathrm{The} \operatorname{Br} \delta$ line is located in a noisy region towards the end of the spectrograph, and shows confusion with night sky lines (OH lines) at $1.945 \mu \mathrm{m} .{ }^{(e)}$ Low- and high-velocity components measured from an aperture of 4 !'7, twice the source size defined in the $\mathrm{CO}(2-1)$ integrated emission. ${ }^{(f)}$ Low-velocity component measured from an aperture of 2'!9. ${ }^{(g)}$ High-velocity component measured from an aperture of 4 '”2.

line intensities were taken by fitting Gaussians to each emission line and are listed in Table 3. For most of the lines, we estimated the Gaussian fit from a velocity range limited to the central channels. Figures $5 \mathrm{~b}$ and $\mathrm{c}$ show the Gaussian fit to the $\mathrm{H}_{2} 1-0 \mathrm{~S}(1)$ and $\mathrm{Br} \gamma$ lines, as well as the residuals of these fittings. Fluxes in Table 3 are not corrected for extinction. Errors in fluxes correspond to the errors obtained from the Gaussian fit, to which we include, as standard measurement errors, the quadratic sum of the standard deviation per channel $\sigma_{\text {chan }}$ (close to the line emission) and the $10 \%$ uncertainty of the SINFONI absolute flux. Velocities are given with respect to the LSR with error-bars estimated from the fitting of $\mathrm{OH}$ sky lines $\left(\sim 1.5-3 \mathrm{~km} \mathrm{~s}^{-1}\right)$. Table 3 also lists the observed line widths (FWHM) derived from the the Gaussian fits, the spectral resolution for each line, and the intrinsic line width (observed value deconvolved by the spectral resolution).

We estimated the extinction in the $K$-band by comparing the $N_{\text {Lyc }}$ values obtained with the thermal radio emission (Sect. 4.1) and that obtained with the $\mathrm{Br} \gamma$ emission. We computed $N_{\text {Lyc }}$ from $\mathrm{Br} \gamma$ assuming case $\mathrm{B}$ recombination, an electron density $n_{\mathrm{e}}=10^{4} \mathrm{~cm}^{-3}$, and an electron temperature of $T_{\mathrm{e}}=10^{4} \mathrm{~K}$ (see Table 6 of Hummer \& Storey 1987). We estimated an extinction for Bry of 0.8 mag (a factor 2), listed in Table 2. Gilbert et al. (2000) reported a slightly higher value of $1.2 \mathrm{mag}$. Their $\mathrm{Br} \gamma$ flux, measured from single-slit spectroscopy, is 1.5 times smaller than ours. Whitmore et al. (2010) reported $E(B-V)$ to be 2.44, and assuming a Draine extinction law, we obtained $A_{K}=0.9$, which agrees with our estimated value. 


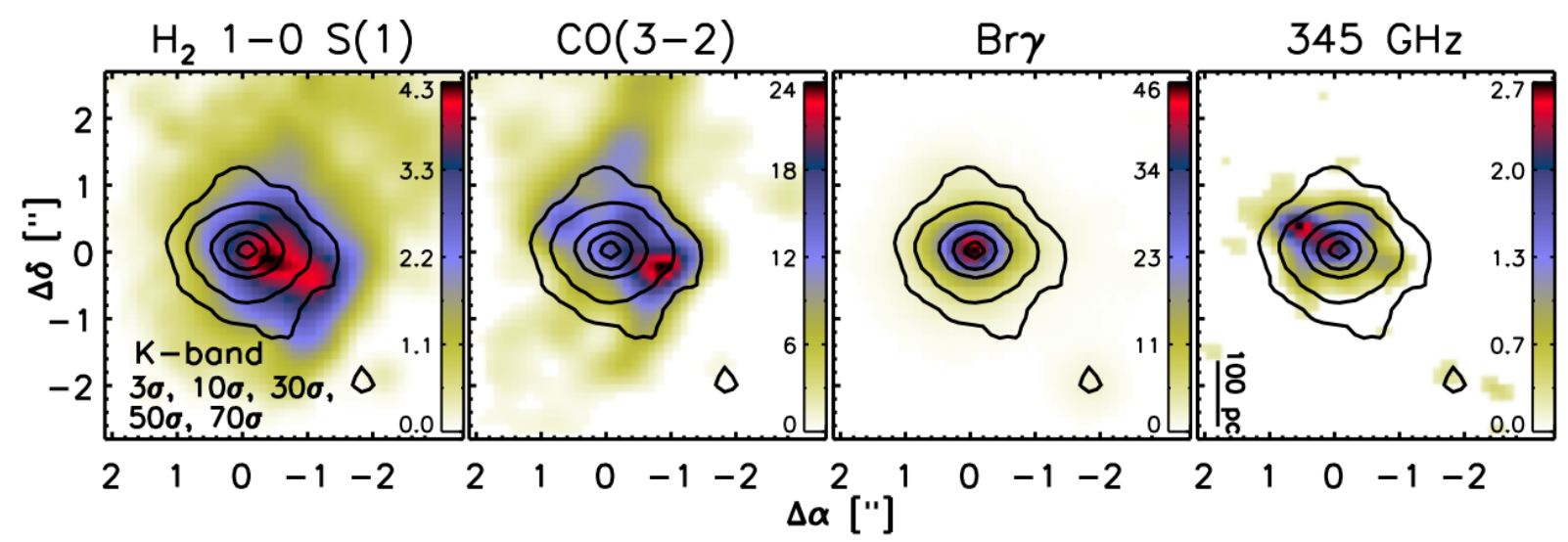

Fig. 6. Intensity maps for SGMC 4/5. From left to right, $K$-band continuum emission contours overlaid on the $\mathrm{H}_{2} 1-0 \mathrm{~S}(1)$ emission, $\mathrm{CO}(3-2)$ emission, Br $\gamma$ emission, and continuum emission at $345 \mathrm{GHz}$. Flux units of the color bars are $\times 10^{-17} \mathrm{erg} \mathrm{s}^{-1} \mathrm{~cm}^{-2}$ for $\mathrm{H}_{2}$ and $\mathrm{Br} \gamma, \mathrm{Jy} \mathrm{beam}{ }^{-1}$ for $\mathrm{CO}(3-2)$, and $\mathrm{mJy}_{\text {beam }}^{-1}$ for the continuum emission. The $1 \sigma$ emission level of the $K$-band continuum emission is $2.05 \times 10^{-20} \mathrm{erg} \mathrm{s}^{-1} \mathrm{~cm}^{-2}$. The peak emission of the $K$-band contours marks the position of the SSC B1, which corresponds to the offset position $(0,0)$. Offset positions are as in Fig. 4.

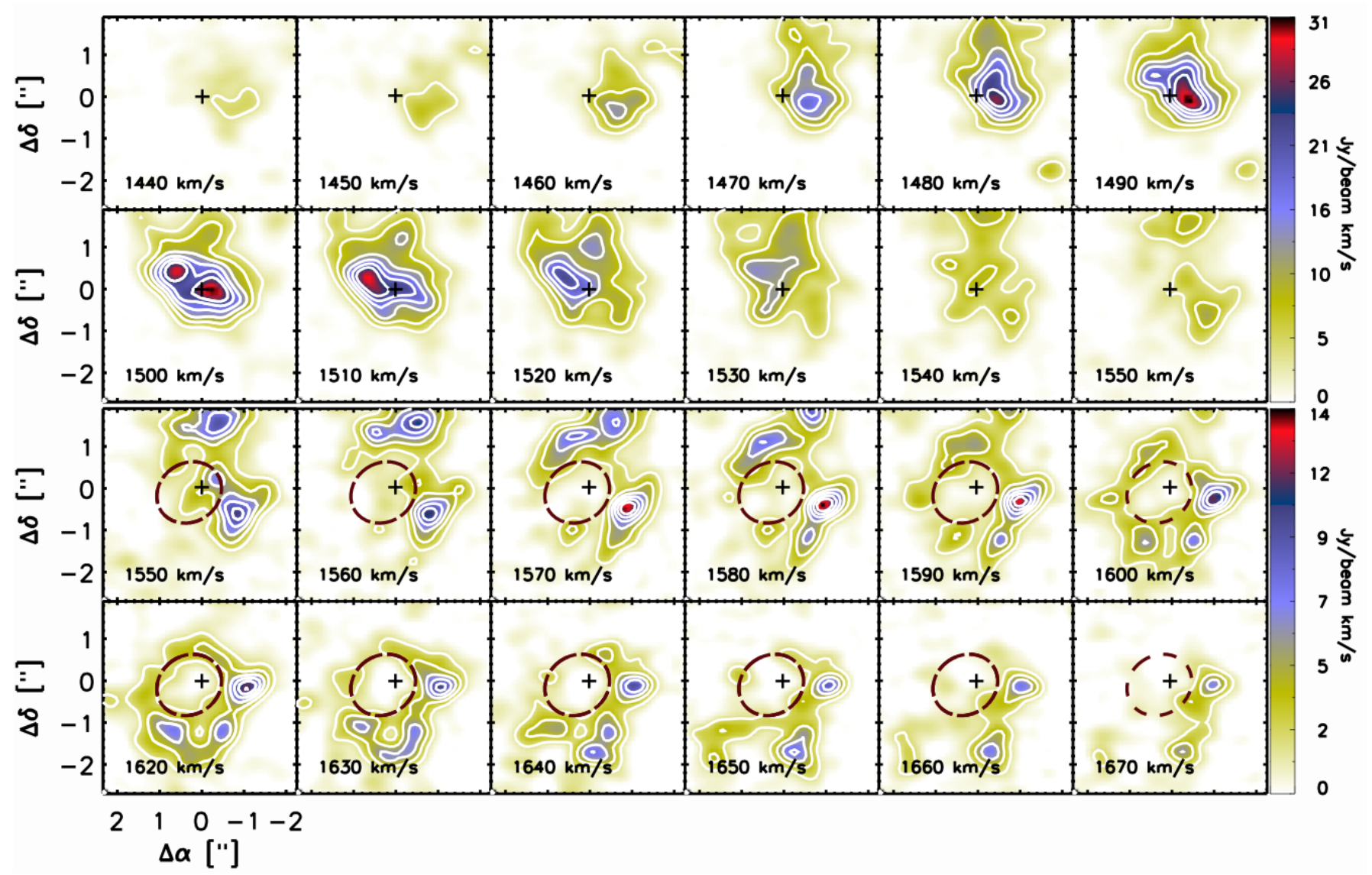

Fig. 7. $\mathrm{CO}(3-2)$ channel maps of SGMC 4/5. The position of SSC B1 is marked with a cross and corresponds to the $(0,0)$ offset position. Top panel: we present channel maps from $1440 \mathrm{~km} \mathrm{~s}^{-1}$ to $1550 \mathrm{~km} \mathrm{~s}^{-1}$ by $10 \mathrm{~km} \mathrm{~s}^{-1}$. Contours start at $10 \sigma$ with a spacing of $10 \sigma$, with $\sigma=$ $0.39 \mathrm{Jy} \mathrm{beam}^{-1} \mathrm{~km} \mathrm{~s}^{-1}$ measured in line-free channels. Bottom panel: CO channel maps from $1550 \mathrm{~km} \mathrm{~s}^{-1}$ to $1670 \mathrm{~km} \mathrm{~s}^{-1} \mathrm{by} 10 \mathrm{~km} \mathrm{~s} \mathrm{~s}^{-1}$. Contours start at $5 \sigma$ with a spacing of $5 \sigma$. Offset positions are as in Fig. 4.

In Fig. 6, we have overlaid the $K$-band continuum emission on the $\mathrm{H}_{2} 1-0 \mathrm{~S}(1), \mathrm{CO}(3-2), \mathrm{Br} \gamma$ line emission, and continuum emission at $345 \mathrm{GHz}$. The peak emission of the $K$-band continuum and $\mathrm{Br} \gamma$ emission coincide; they define the position of the SSC. The peak in the dust continuum emission at $345 \mathrm{GHz}$ coincides with a water maser discovered by Brogan et al. (2010). Figure 4 displays the continuum emission with the water maser subtracted, its emission peak coincides with the position of the
$\mathrm{SSC}$. The molecular gas, that is, $\mathrm{H}_{2}$ and $\mathrm{CO}$ emission, is more extended than the ionized gas. Neither $\mathrm{H}_{2}$ or $\mathrm{CO}$ peaks exactly at the position of the SSC. Figure 7 presents the $\mathrm{CO}(3-2)$ channel maps from 1440 to $1670 \mathrm{~km} \mathrm{~s}^{-1}$. From these channel maps, we can distinguish two spatially and spectrally separated components. Figure 8 presents the spatial extent (zero moment map) and first moment map of these components. The left top and bottom panels show the $\mathrm{CO}(3-2)$ emission integrated over the 

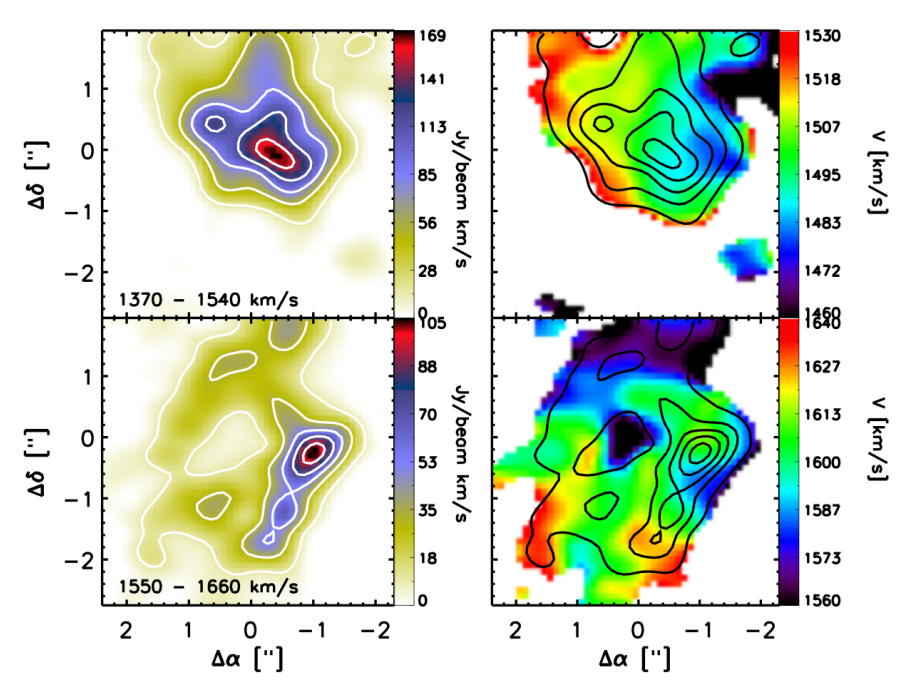

Fig. 8. $\mathrm{CO}(3-2)$ velocity components in SGMC 4/5. Left panels: $\mathrm{CO}$ emission integrated between $1370 \mathrm{~km} \mathrm{~s}^{-1}$ and $1540 \mathrm{~km} \mathrm{~s}^{-1}$ (top) and $1550 \mathrm{~km} \mathrm{~s}^{-1}$ and $1660 \mathrm{~km} \mathrm{~s}^{-1}$ (bottom). Contours represent the $10 \%$, $30 \%, 50 \%, 70 \%$, and $90 \%$ of the peak emission. Right panels: first moment of both velocity components. Contours are the same as in the left panel. Offset positions are as in Fig. 4.

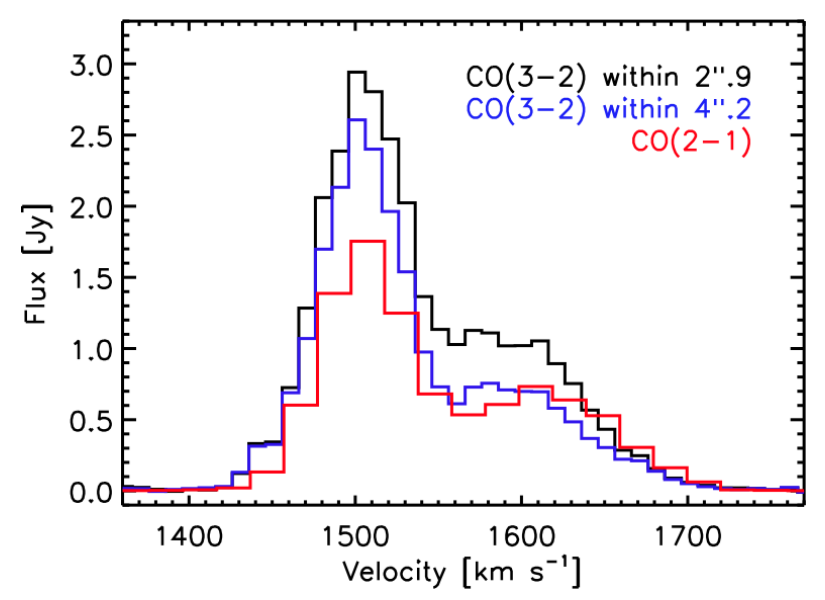

Fig. 9. $\mathrm{CO}(3-2)$ and $\mathrm{CO}(2-1)$ spectra associated with SSC B1. For the CO $J=3-2$ transition, we present the spectra measured with two apertures, 2 ".9 and 4 ".2, to characterize the two velocity components observed in the channel maps in Fig. 7.

velocity ranges $1370-1540 \mathrm{~km} \mathrm{~s}^{-1}$ and $1550-1660 \mathrm{~km} \mathrm{~s}^{-1}$, respectively. There is a spatial offset between the two velocity components. The low-velocity emission is mainly associated with the SSC, while the higher velocity component seems to surround the cluster in a bubble-like shape structure, having the spatial extent of the SGMC. This bubble-like shape was already highlighted by Whitmore et al. (2014, see their Fig. 4). The first moment maps show that the low-velocity component has a velocity gradient while the high-velocity component is almost at the same velocity.

We measure the $\mathrm{CO}(3-2)$ line flux of the low-velocity component within an aperture diameter of $2^{\prime \prime} .9$, twice the observed FWHM size defined in the $\mathrm{CO}(3-2)$ emission integrated between $1370 \mathrm{~km} \mathrm{~s}^{-1}$ and $1540 \mathrm{~km} \mathrm{~s}^{-1}$ (see top-left figure in Fig. 8), and centered on the peak emission of this component. The resulting spectrum is plotted with a black line in Fig. 9. It has a non-Gaussian profile. Line parameters were estimated by fitting 2 Gaussian curves, but only those of the low-velocity
Table 4. Parameters of the two CO velocity components.

\begin{tabular}{lcc}
\hline \hline & Low-velocity & High-velocity \\
\hline$M_{\mathrm{mol}}$ & $5.1 \pm 0.2 \times 10^{7} M_{\odot}$ & $3.4 \pm 0.2 \times 10^{7} M_{\odot}$ \\
$\mathrm{CO}(3-2)$ & $\sim 0.7$ & $\sim 0.6$ \\
$\mathrm{CO}(2-1)$ & & $<0.18$ \\
\hline $\mathrm{H}_{2} 2-1 \mathrm{~S}(1)$ & $0.32 \pm 0.04$ & $<\mathrm{H}(1)$ \\
\hline
\end{tabular}

component are listed in Table 3. Figure 8 shows that the highvelocity component has a larger spatial extent. We measure the $\mathrm{CO}(3-2)$ line flux of the high-velocity component within an aperture of 4 ".2 diameter, estimated from the size of the $\mathrm{CO}(3-2)$ emission integrated in the high-velocity channels. This size coincides with that measured in the $\mathrm{H}_{2} 1-0 \mathrm{~S}(1)$. The result is plotted with a blue line in Fig. 9. The parameters listed in Table 3 for the high-velocity $\mathrm{CO}(3-2)$ emission component are derived from the fit of this spectrum with two Gaussian curves. To quantify the $\mathrm{CO}(2-1)$ emission, we used an aperture diameter of 4 ".7 (red line in Fig. 9), size computed from the total $\mathrm{CO}(2-1)$ integrated emission. We fit the emission with two Gaussian curves. Table 3 lists their line parameters. We do not subtract the water maser contribution to the $\mathrm{CO}$ emission, as we did for the continuum emission (Sect. 4.2), because the velocity structure is very complex, making it difficult to separate the contribution of the water maser from the total $\mathrm{CO}$ emission. However, this shortcoming does not affect the conclusions of this paper. Systematic uncertainties in the $\mathrm{CO}$-to- $\mathrm{H}_{2}$ conversion factor are much larger than the potential contribution of the maser to the $\mathrm{CO}$ emission.

Figure 9 displays the $\mathrm{CO}(3-2)$ spectra from both apertures mentioned above as well as the $\mathrm{CO}(2-1)$ spectra. Both $\mathrm{CO}$ transitions show the two velocity components observed in the channel maps. From the Gaussian fit, we find that the high-velocity component is almost twice wider than the low-velocity component. In Table 4, we list the $\mathrm{CO}(3-2)$-to- $\mathrm{CO}(2-1)$ line ratios for both components. The ratio was measured within an aperture of 4 !.7 defined by the $\mathrm{CO}(2-1)$ size and after imaging the $\mathrm{CO}(3-2)$ observations using the $\mathrm{CO}(2-1)$ synthesized beam as restored beam during the imaging process (CLEAN algorithm in CASA). The intensity ratio between the $J=3-2$ and 2-1 CO transitions depends on the gas temperature and density as discussed by Schulz et al. (2007) in the modeling of their single dish observations of the Antennae. We do not see a significant difference in the excitation of the gas between the low- and high-velocity components.

\subsection{Mass of the GMC}

We estimate the mass of the GMC, associated with SSC B1, from the continuum emission at $345 \mathrm{GHz}$. We approximate the spectral dependence on the dust emission with a gray body. We assume that the dust properties in the Antennae overlap region are the same as those in the Galaxy. Scoville et al. (2014) discuss the use of the Galactic values from Planck observations (Planck Collaboration XXV 2011) to estimate the millimetric masses in local starburst galaxies. They also show that dust temperatures between 15 and $30 \mathrm{~K}$ are typically found in starbursts (see also Dunne et al. 2011), even in extreme cases such as Arp 220, where the dust temperature does not exceed $45 \mathrm{~K}$. In the Antennae, metallicities of approximately the solar one are observed towards several SSCs (Bastian et al. 2009). We assume a typical dust temperature of $25 \mathrm{~K}$ and 
the emissivity per hydrogen measured in the Taurus molecular cloud by Planck Collaboration XXV (2011), which is similar to that obtained for starburst galaxies (Scoville et al. 2014). Using Eq. (8) in Herrera et al. (2013), we derive the mass of the giant molecular cloud of $M_{\mathrm{GMC}}=3.3 \pm 0.1 \times 10^{7} M_{\odot}$, listed in Table 2. The error is estimated as the $1 \sigma$ standard deviation of the continuum image measured with the same aperture over nearby regions free of sources. Uncertainty on the dust temperature implies an uncertainty of a factor 2 in the mass (if $T_{\mathrm{d}}$ is $15 \mathrm{~K}$, the mass will be twice the estimated value, while for $T_{\mathrm{d}}=45 \mathrm{~K}$, the mass will be half the estimated value). The gas column density $N_{\mathrm{H}}=4.5 \pm 0.3 \times 10^{23} \mathrm{H} \mathrm{cm}^{-2}$ is estimated assuming spherical symmetry.

The virial mass, assuming a spherical cloud with a density profile $\propto r^{-1}$, can be written as $M_{\text {vir }}=190 \Delta v^{2} R$ (MacLaren et al. 1988). We estimate the virial mass of the low-velocity component to be $\sim 4 \times 10^{7} M_{\odot}$. We also estimate the molecular gas mass from the observed $\mathrm{CO}(3-2)$ emission. We assume a $\mathrm{CO}$-to- $\mathrm{H}_{2}$ conversion factor of $0.6 \times 10^{20} \mathrm{H}_{2} \mathrm{~cm}^{-2}\left(\mathrm{~K} \mathrm{~km} \mathrm{~s}^{-1}\right)^{-1}$, estimated by Zhu et al. (2003) for the overlap region at scales of $\sim 1.5 \mathrm{kpc}$ and comparable to the value estimated by Kamenetzky et al. (2014) using CO modeling at $4.5 \mathrm{kpc}$ scales (Herschel data) in the overlap region. This value is also comparable to the typical value found for starburst galaxies, ultraluminous infrared galaxies and towards the Galactic center (for a detailed discussion about the uncertainties on this factor see Bolatto et al. 2013). Other authors have estimated the conversion factor to be similar to the Galactic one (e.g., Wilson et al. 2003; Schulz et al. 2007). In this paper we choose to use a starburst-like value, which gives us a good agreement with our other measurements of the molecular gas (see below). We also assume a $\mathrm{CO}(3-2)$-to- $\mathrm{CO}(1-0)$ ratio of $\sim 0.6$, as measured by Schulz et al. (2007) with single dish data in the overlap region and Ueda et al. (2012) with interferometric observations at 500 pc scales. Using Eq. (3) from Bolatto et al. (2013), we estimate $M_{\text {mol }}$ to be $5.1 \times 10^{7} M_{\odot}$. Using the Galactic CO-to- $\mathrm{H}_{2}$ conversion factor of $2 \times 10^{20} \mathrm{H}_{2} \mathrm{~cm}^{-2}$ $\left(\mathrm{K} \mathrm{km} \mathrm{s}^{-1}\right)^{-1}$ (Solomon et al. 1987), the mass would be more than three times higher. The estimated $M_{\text {mol }}$ value agrees with the value estimated from the continuum emission and the virial theorem, indicating that the $\mathrm{CO}-$ to- $\mathrm{H}_{2}$ conversion factor estimated at $1.5 \mathrm{kpc}$ scales by Zhu et al. (2003) is also adequate for SGMC 4/5. The measured flux from the high-velocity component yields a mass of $3.4 \times 10^{7} M_{\odot}$. We list these values in Table 4.

\section{Physical structure of the surrounding matter}

In this section, we describe the physical structure of the molecular gas surrounding SSC B1 which we associate with the narrow velocity component of the $\mathrm{CO}$ spectrum in Fig. 9 and Table 3. We measure the molecular gas pressure and conclude that there is no trapping of IR light within the internal cavity (Sect. 5.1). In Sect. 5.2, we estimate the hot plasma pressure and find that the gas around SSC B1 is distributed in an inhomogeneous, already broken shell.

\subsection{Gas pressure in molecular gas}

Radiation pressure $P_{\text {rad }}$ is the pressure exerted by the stellar radiation over the molecular gas that surrounds the central star cluster. We quantify the radiation pressure in the molecular gas surrounding SSC B1. We employ the expression from
Murray et al. (2010), which includes the opacity of the shell,

$P_{\mathrm{rad}}=\left(1+\left\langle\tau_{\mathrm{rad}}\right\rangle\right) \frac{L_{\mathrm{cl}}}{4 \pi R_{\mathrm{in}}^{2} c}$,

where $L_{\mathrm{cl}}$ is the luminosity of the cluster, $R_{\mathrm{in}}$ is the H II radius, and $\left\langle\tau_{\text {rad }}\right\rangle$ accounts for the trapping of the IR photons in the cloud $\left(\left\langle\tau_{\text {rad }}\right\rangle \geq 0\right)$. IR photons originate from UV and visible photons emitted by the cluster, which are absorbed by the dust grains in the cloud, and re-emitted in the IR. While the shell surrounding the cluster is closed and opaque, the IR photons are trapped within the shell, interacting more than once with the dust grains in the shell. The radiation pressure is estimated from the bolometric luminosity emitted by the SSC, $L_{\mathrm{cl}}=5.3 \times 10^{9} L_{\odot}$ and the $\mathrm{H}$ II region radius $R_{\text {in }}=35 \mathrm{pc}(\mathrm{Br} \gamma$ size $)$. We compute

$$
\frac{P_{\mathrm{rad}}}{k_{\mathrm{B}}}=3.4 \times 10^{7} \times\left(1+\left\langle\tau_{\mathrm{rad}}\right\rangle\right) \times\left(\frac{[35 \mathrm{pc}]}{R_{\mathrm{in}}}\right)^{2} \mathrm{~K} \mathrm{~cm}^{-3}
$$

There are large uncertainties for the age and mass of the cluster (Sect. 4.1) and the value given in Eq. (2) is a lower limit obtained for the lowest value of $L_{\mathrm{cl}}$ in Table 2. If we use the $L_{\mathrm{cl}}$ estimated for $3.5 \mathrm{Myr}$ and $1.5 \times 10^{7} M_{\odot}$, the age and mass computed from the SINFONI data, the radiation pressure is three times higher. In SSC B1, the moderate value of the effective extinction in the $K$-band (see Table 2) preclude large values of $\left\langle\tau_{\text {rad }}\right\rangle$.

The observational results presented in Sect. 4 allow us to model the physical environment of SSC B1 (see Appendix A for more details). We model the H II region surrounding the SSC as an ionized nebula with dust grains, with incident radiation field of that from the SSC. For different sizes of the internal cavity, we solve the radiative transfer equation within the H II region and measure the outward radiation field and the pressure exerted at the surface of the PDR. We assume that the gas pressure is set by the radiation pressure and compare our grid of $P_{\text {rad }}$ and radiation field values with the outputs of the PDR Meudon code (Le Bourlot et al. 2012), which predicts molecular line intensities from PDRs. We chose the standard isobaric model with fixed density. These models are detailed in Appendix A. We find that PDR models with radiation fields in a range of $\chi \sim 10^{3}-$ $10^{4}$ times the mean radiation field in the solar neighborhood and a gas pressure of $3 \times 10^{7}-10^{8} \mathrm{~K} \mathrm{~cm}^{-3}$ are needed to account for the observed $\mathrm{H}_{2}$ 1-0/2-1 S(1) line emission (see Fig. A.1). The value of the radiation field is consistent with that estimated by Gilbert et al. (2000) from the comparison of PDR models and several near-IR $\mathrm{H}_{2}$ line intensities (obtained with single-slit spectroscopy towards SSC B1). The estimated gas pressure of $3 \times 10^{7}-10^{8} \mathrm{~K} \mathrm{~cm}^{-3}$ agrees with the measured molecular gas pressure (Eq. (2)), supporting low values of $\left\langle\tau_{\text {rad }}\right\rangle$. There is no significant trapping of the IR photons within the cavity of the molecular gas surrounding SSC B1.

\subsection{Gas pressure in the hot gas}

SSC B1 was detected as a compact X-ray source by Chandra (source \#88 in Table 5 of Zezas et al. 2006). Its X-ray luminosity, integrated between $0.1-10 \mathrm{keV}$, is $L_{\mathrm{X}}=1.9 \pm 0.2 \times 10^{38} \mathrm{erg} \mathrm{s}^{-1}$. This X-ray luminosity can be used to estimate the electronic density $n_{\mathrm{e}}$ and thus the hot gas pressure.

$L_{\mathrm{X}}=\epsilon(T) \int n_{\mathrm{e}}^{2} \mathrm{~d} l \times A \simeq 4 \pi \epsilon(T) n_{\mathrm{e}}^{2} R_{\mathrm{in}}^{3}$,

where $\epsilon(T)=3 \times 10^{-23} \mathrm{erg} \mathrm{s}^{-1} \mathrm{~cm}^{3}$ is the emission coefficient (assuming a temperature of $T=10^{7} \mathrm{~K}$, from Fig. B. 1 in 
Guillard et al. 2009), $E M=\int n_{\mathrm{e}}^{2} \mathrm{~d} l \simeq n_{\mathrm{e}}^{2} \times R_{\text {in }}$ is the emission measure, and $A=4 \pi R_{\text {in }}^{2}$ is the area where the pressure is exerted. For the observed value of $L_{\mathrm{X}}$, we find an electron density of $n_{\mathrm{e}}=0.6 \times\left([35 \mathrm{pc}] / R_{\mathrm{in}}\right)^{3 / 2} \mathrm{~cm}^{-3}$. The pressure of the hot plasma is $P_{\text {hot, } \mathrm{X}}=1.9 n_{\mathrm{e}} k_{\mathrm{B}} T$. For a temperature of $T=10^{7} \mathrm{~K}$, the hot plasma pressure is

$$
\frac{P_{\mathrm{hot}, \mathrm{X}}}{k_{\mathrm{B}}}=1.2 \pm 0.4 \times 10^{7} \times\left(\frac{[35 \mathrm{pc}]}{R_{\mathrm{in}}}\right)^{3 / 2} \mathrm{~K} \mathrm{~cm}^{-3} \text {. }
$$

This value is a lower limit because we did not correct the X-ray luminosity for absorption by the shell. Figure 1 in Ryter (1996) shows that the absorption in the X-ray by the gas is comparable to the extinction in the $K$-band and it is thus a factor of approximately 2 , increasing $P_{\text {hot, } \mathrm{X}}$ by a factor 1.4 .

We find that the hot gas pressure is at least three times weaker than the pressure of the molecular gas. This difference indicates that the hot gas pressure is not dynamically important. The hot plasma produced by shocks driven by stellar winds (Castor et al. 1975 ) is not trapped within a closed shell. If the mechanical energy from stellar winds over 1 Myr (age of SSC B1) was confined within a closed shell of interstellar matter, then the gas pressure in the hot gas would be more than 50 times higher than that estimated in Eq. (4) (if we use the age and masses estimated from the $\operatorname{Br} \gamma \mathrm{EW}$ and radio $N_{\mathrm{Lyc}}$, then it would be three orders of magnitude higher). This result is consistent with our finding that $\left\langle\tau_{\text {rad }}\right\rangle$ is negligible. It also implies that the molecular gas interfaces heated by the SSC must be distributed over a range of distances. The estimated molecular radius of $\sim 50 \mathrm{pc}$ (Table 2) is a mean value.

\section{Radiation pressure feedback}

In this section we discuss the role of radiation pressure as a stellar feedback process in SSC B1.

\subsection{Acceleration of the gas by stellar feedback}

In Sect. 5, we show that radiation pressure is the dominant pressure on the molecular gas closest to the cluster, in agreement with previous studies in massive clusters (e.g., Krumholz \& Matzner 2009; Murray et al. 2010; Lopez et al. 2011). We compare this outward pressure with the gravitational attraction exerted by the central cluster and the mass of the cloud itself. For a spherical distribution of the matter, we estimate the inward force per unit surface as,

$$
\begin{aligned}
& P_{\text {grav }}=\frac{1}{4 \pi r^{2}}\left(\frac{G M M_{\mathrm{cl}}}{r^{2}}+\frac{G M^{2}}{2 r^{2}}\right) \\
& \frac{P_{\text {grav }}}{k_{\mathrm{B}}}=1.6 \times 10^{8}\left(\frac{52 \mathrm{pc}}{r}\right)^{4} \mathrm{~K} \mathrm{~cm}^{-3},
\end{aligned}
$$

where $r$ and $M$ are the radius and mass of the GMC, and $M_{\mathrm{cl}}$ is the mass of the SSC taken from Table 2. We note that the factor 2 in the uncertainty on the GMC mass (see Sect. 4.4) introduces an uncertainty of a factor 3 in $P_{\text {grav }}$. The radiation pressure, as estimated in Eq. (2), is weaker than the gravitational force. It is not strong enough to push away the molecular gas associated with SSC B1.

In the following section, we speculate that most (or at least a significant part) of the pre-cluster gas has already been blown away and that the high-velocity component is tracing outflowing molecular gas. From the ALMA observations, Whitmore et al. (2014) also identify this component as a super-bubble. Earlier, this gas would have been accelerated by radiation pressure when it was much higher than today.

\subsection{Outflowing gas}

The momentum of the outflowing gas can be estimated from the molecular mass in the high-velocity component of $\mathrm{CO}(3-2)$ (taken from Table 4) and the expansion velocity of that gas $v_{\text {exp }}$. The latter value can be estimated using different assumptions that we discuss in Sect. 7.1. We find that the expansion velocity of the gas is $v_{\exp } \sim 80 \mathrm{~km} \mathrm{~s}^{-1}$. It is larger than the escape velocity from the cluster (using the size measured in the $K$-band continuum emission of $66 \mathrm{pc}$ ), which is $v_{\mathrm{esc}}=\sqrt{2 G M_{\mathrm{cl}} / R_{\mathrm{cl}}}=$ $42 \mathrm{~km} \mathrm{~s}^{-1}$. Uncertainties on the expansion velocity come from the unknown 3D geometry of the gas and from the mass of cluster.

To test the hypothesis that this gas was accelerated by radiation pressure over a time scale of $t_{\text {feedback }}$, we write,

$\frac{L_{\mathrm{cl}}}{c}\left(1+\tau_{\mathrm{rad}}\right) t_{\mathrm{feedback}}=M_{\mathrm{high}} v_{\mathrm{exp}}$,

where

$t_{\text {feedback }} \simeq R_{\text {high }} / v_{\text {exp }} \simeq 1.2 \mathrm{Myr}$,

with $R_{\text {high }} \sim 100 \mathrm{pc}$, the mean size of the bubble-like shape structure observed in this component (Fig. 7), and $\tau_{\text {rad }}$ refers to the effective opacity of the cloud to radiation (Sect. 5.1). From Eq. (6), we can constrain the value of $\tau_{\text {rad }}$ as:

$\left(1+\tau_{\mathrm{rad}}\right)=\frac{M_{\mathrm{high}} v_{\mathrm{exp}}^{2}}{\frac{L_{\mathrm{cl}}}{c} R_{\mathrm{high}}} \simeq 21 \times\left(\frac{M_{\mathrm{high}}}{3.4 \times 10^{7} M_{\odot}}\right)$.

This value is higher than that observed today, but within plausible values earlier in the cluster evolution when the parent cloud was not yet disrupted. Indeed, in the models by Murray et al. (2010), $\tau_{\text {rad }}$ reaches values of several tens at the very beginning of the cluster evolution. Smaller values for $\tau_{\text {rad }}(\sim 6)$ and $v_{\exp }$ $\left(\sim 60 \mathrm{~km} \mathrm{~s}^{-1}\right)$ are found if we use the age estimated from the $\mathrm{Br} \gamma$ EW $\left(L_{\mathrm{cl}}\right.$ is higher, see Table 2$)$.

We estimate a lower limit for the SFE within $100 \mathrm{pc}$ from the cluster of $17 \%$, considering that the high-velocity component may include not only natal gas but surrounding gas that did not participate in the cluster formation. We estimate the outflow rate of the parent molecular cloud to be $\dot{M}_{\text {outflow }}=M_{\text {high }} / t_{\text {feedback }} \simeq$ $30 M_{\odot} / \mathrm{yr}$.

The broad velocity component is also observed in the $\mathrm{H}_{2}$ 1-0 $\mathrm{S}(1)$ emission. The left panel in Fig. 10 presents the spectra for the $v=1-0$ and 2-1 $\mathrm{S}(1) \mathrm{H}_{2}$ line emission integrated within an aperture of $4 . .2$ (Sect. 4.2). The $v=2-1$ line can be fitted with a single Gaussian curve (red-dashed line in Fig. 10) while $v=1-0$ requires two Gaussian components (blue line in Fig. 10). We fit the $v=2-1$ line and use the estimated center velocity as a fixed parameter to fit the low-velocity component of the $v=1-0$ emission. The right panel of Fig. 10 shows the low- and high-velocity components, observed in $v=1-0$, in red and black contours, respectively, overlaid on the $\mathrm{CO}(3-2)$ highvelocity component. The extent of the high-velocity components seen in $\mathrm{CO}(3-2)$ and $\mathrm{H}_{2} 1-0 \mathrm{~S}(1)$ are similar. Comparing Fig. 10 with Fig. 8, we find that the extent of the low-velocity components seen in $\mathrm{CO}(3-2)$ and $\mathrm{H}_{2} 1-0 \mathrm{~S}(1)$ are also similar. 

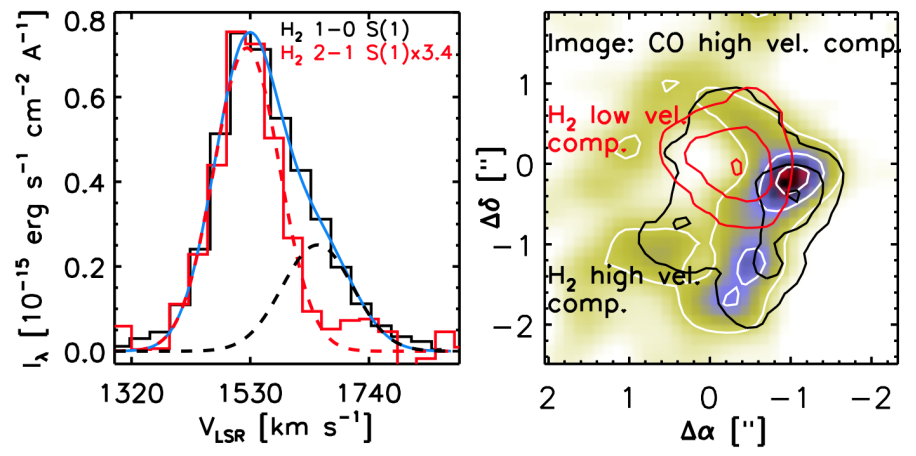

Fig. 10. $\mathrm{H}_{2} 2-1 / 1-0 \mathrm{~S}(1)$ ratio in SGMC $4 / 5$. The left panel shows the spectra for the two near-IR $\mathrm{H}_{2}$ lines, $\mathrm{H}_{2}$ 1-0 S(1) in solid-black and $\mathrm{H}_{2} 2-1 \mathrm{~S}(1)$ in solid-red line scaled by a factor of 3.4. The dashed-red line is the Gaussian fit for the $\mathrm{H}_{2}$ 2-1 S(1) line, while the blue-solid line is the fit for the $\mathrm{H}_{2} 1-0 \mathrm{~S}(1)$ emission that corresponds to the addition of two Gaussian curves (the red and black dashed lines). The color image and white contours in the right panel show the integrated intensity of the high-velocity component observed in $\mathrm{CO}(3-2)$. Red and black contours are the $\mathrm{H}_{2}$ emission integrated in $1300-1512 \mathrm{~km} \mathrm{~s}^{-1}$ (low-velocity component) and 1580-1760 $\mathrm{km} \mathrm{s}^{-1}$ (high-velocity component), respectively. Offset positions are as in Fig. 4.

The $R_{\mathrm{S}(1)}=\mathrm{H}_{2} 2-1 / 1-0 \mathrm{~S}(1)$ ratio is commonly used to disentangle between $\mathrm{H}_{2}$ gas heated by collisions or UV radiation. On the one hand, shock excitation is collisional and only low- $v$ $\mathrm{H}_{2}$ levels are populated because the temperature of molecular gas is, at most, a few $1000 \mathrm{~K}$; for higher temperatures the $\mathrm{H}_{2}$ molecule is destroyed in collisions. On the other hand, $\mathrm{H}_{2}$ excitation through UV pumping and fluorescence populates both high- and low- $v$ states (e.g., Herrera et al. 2011). We find $R_{\mathrm{S}(1)}=$ $0.32 \pm 0.04$ for the low-velocity component, and estimate an upper limit of 0.18 for the high-velocity component using the ratio between the $3 \sigma$ emission for $v=2-1$ ( $\sigma$ measured in the linefree channels) and the peak emission of the high-velocity component seen in $v=1-0$. These values are listed in Table 4 . The former ratio can be accounted for by UV heating of the gas in PDRs (see Sect. 5.1 and Appendix A). The latter ratio can be observed towards PDRs with high radiation fields $\left(>10^{4}\right)$ and gas pressure $\left(\simeq 10^{8} \mathrm{~K} \mathrm{~cm}^{-3}\right)$ (see Fig. A.1). However, since the highvelocity component is extended (a few $100 \mathrm{pc}$ ) and there is no evidence for extended massive stellar population, we favor shocks as the main gas heating mechanism. Values in the range $0.1-0.2$ are reproduced in J- and C-shock models (Kristensen 2007) for gas with densities in the range $10^{4}-10^{6} \mathrm{~cm}^{-3}$ and shock velocities from 15 to $50 \mathrm{~km} \mathrm{~s}^{-1}$. This supports the idea that the highvelocity component is tracing outflowing gas. This interpretation indicates that the two velocity components have different excitation mechanisms.

Evidence of high-velocity ionized gas can be observed in the $\mathrm{Br} \gamma$ line emission. Figure 11 presents the channel maps of the $\mathrm{Br} \gamma$ line emission overlaid on the $\mathrm{CO}(3-2)$ line emission. At the velocities of the low-velocity component $\left(<1600 \mathrm{~km} \mathrm{~s}^{-1}\right)$, the $\mathrm{Br} \gamma$ emission is symmetrical. An excess of ionized gas emission, where the $\mathrm{CO}(3-2)$ has a low column density, is observed at velocities of $1600 \sim 1700 \mathrm{~km} \mathrm{~s}^{-1}$, that we interpret as evidence of escaping ionized gas.

\section{Possible scenario}

In our interpretation, it is too late to witness the first stages of the disruption of the parent molecular cloud by stellar feedback, when the parent cloud was still a bound cloud. Nevertheless, the

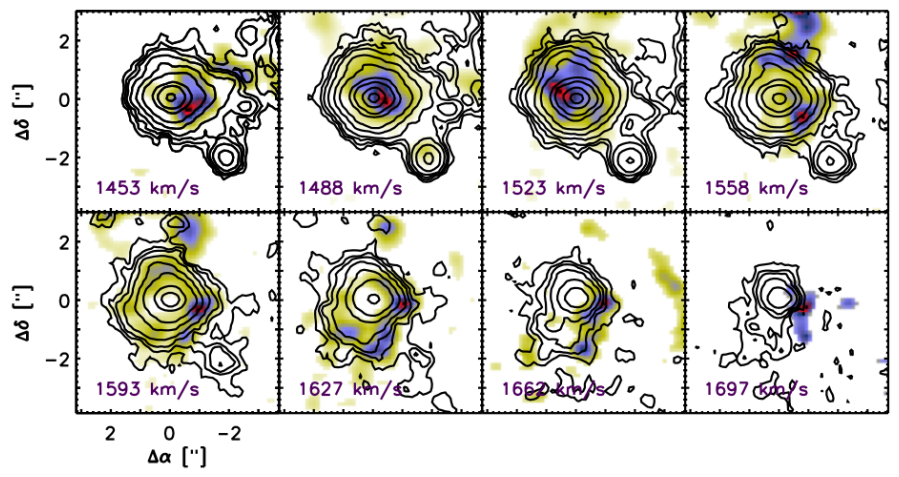

Fig. 11. Contours are the channel maps of the ionized gas traced by $\mathrm{Br} \gamma$ overlaid on the $\mathrm{CO}(3-2)$ emission interpolated to the velocity of the SINFONI observations.

high-velocity component may be tracing the outflowing gas from this cloud. In the following, we discuss this interpretation and its caveats.

\subsection{Disruption of the parent cloud and outflowing gas}

We propose that radiation pressure was the main mechanism disrupting the parent molecular cloud over a short timescale. At the beginning of the SSC evolution, radiation pressure was much higher than today due to the high density and opacity of the parent cloud. The IR photons from the dust heated by the UV photons from the cluster were highly trapped in the cloud implying a high $\tau_{\text {rad }}$ value and radiation pressure (see Eq. (1)). The momentum transfer rapidly accelerates the surrounding gas to velocities sufficiently high to escape from the cluster. Murray et al. (2010) modeled this disruption process for a source with similar characteristics (i.e., GMC mass and size, and star cluster mass), finding that the disruption occurs in less than $1 \mathrm{Myr}$.

Within this scenario, the high-velocity component is tracing outflowing gas from the parent cloud. The expansion time scale of 1.2 Myr, computed in Sect. 6.2, agrees with the model value from Murray et al. (2010). It is comparable to the age of the cluster (1-3.5 Myr), which makes this scenario of cloud disruption plausible. The bottom panel of Fig. 7 shows the channel maps of this component, which surrounds SSC B1 in a bubble-like shape structure with an inner radius of approximately $1^{\prime \prime}(\sim 100 \mathrm{pc})$. Similar molecular gas morphology and kinematics are observed in the starburst galaxies M82 and NGC 253 (Weiß et al. 1999; Sakamoto et al. 2006), where the data have been interpreted as evidence of expanding molecular super-bubbles. Evidence of expanding bubbles has also been observed for ionized gas (e.g., in M83, Hollyhead et al. 2015).

In Fig. 12, we show the $\mathrm{CO}(3-2)$ emission averaged within concentric annuli centered on SSC B1 up to a distance of 1".8 from the cluster (azimuthally averaged $\mathrm{CO}(3-2)$ emission). For a closed, spherical and homogeneous expanding shell, the azimuthally averaged gas emission should show a semi-ellipse with constant flux. This is not the case for the super-bubble in SSC B1. The central velocity of the cluster is difficult to determine. We can approximate this velocity in two ways. First, we assume that the $\mathrm{Br} \gamma$ emission is symmetrical (no obscuration by dust) and therefore use the $\mathrm{Br} \gamma$ velocity as a proxy for the cluster velocity. In this case, we approximate the velocity expansion of the bubble as the difference between the $\operatorname{Br} \gamma$ velocity and the velocity of the high-velocity component, $\sim 80 \mathrm{~km} \mathrm{~s}^{-1}$. Figure 12 shows a cyan dashed-line semi-ellipse to illustrate this scenario. 


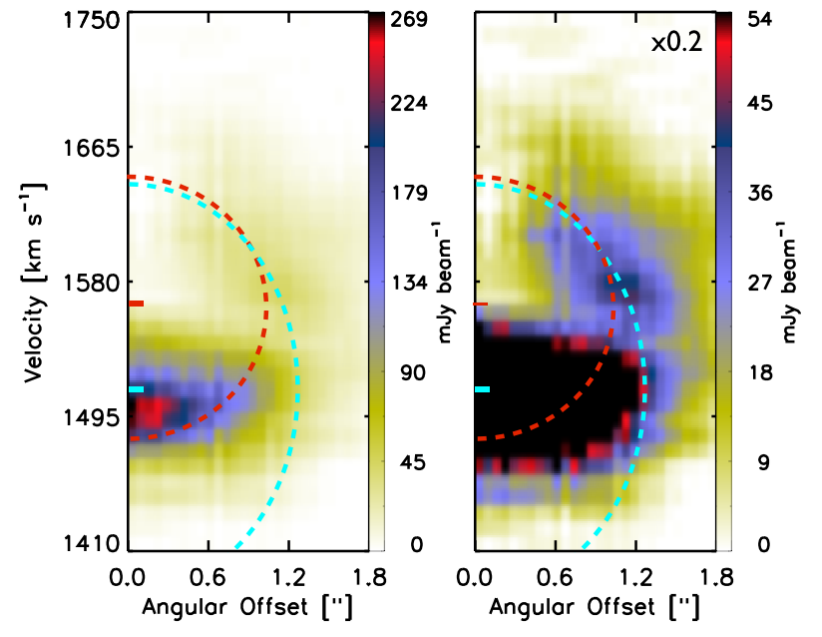

Fig. 12. Azimuthally averaged $\mathrm{CO}(3-2)$ emission, around the position of SSC B1 up to a maximum radius of 1":8. The left panel shows the total emission and the right panel shows the emission clipped up to a maximum value of $20 \%$ of the peak emission. The semi-ellipse in dashed-red line highlights the expanding shell assuming a central velocity of $\sim 1565 \mathrm{~km} \mathrm{~s}^{-1}$ marked with a red tick on the velocity axis, while for the dashed-cyan line, the central velocity is equal to that of the $\mathrm{Br} \gamma$ emission.

The size of the ellipse was chosen to fit the CO high-velocity emission. In this scenario, the $\mathrm{CO}$ data do not show any evidence of blue-shifted gas and we have to assume that the $\mathrm{CO}$ shell is not symmetric. Second, we estimate the velocity of the cluster fitting the $\mathrm{CO}$ azimuthally averaged emission. The red dashed-line in Fig. 12 shows the best fit for the $\mathrm{CO}$ emission (low- and high-velocity components), with a central velocity of $\sim 1565 \mathrm{~km} \mathrm{~s}^{-1}$, which would be tracing the expanding bubble. In this scenario, the central velocity of the ellipse is approximately $50 \mathrm{~km} \mathrm{~s}^{-1}$ larger than that of the compact Br $\gamma$ emission seen towards the SSC. This difference with the $\mathrm{Br} \gamma$ velocity may imply that the red-shifted $\mathrm{Br} \gamma$ gas is obscured and we only observe the gas that is the closest to us. An expanding ionized bubble with asymmetrical emission has been observed towards a young (6 Myr) and massive $\left(10^{7} M_{\odot}\right)$ SSC in a blue compact galaxy (Östlin et al. 2007).

\subsection{The molecular gas surrounding SSC B1}

If the parent cloud was disrupted early in the cluster evolution, we are curious as to why we see a $\mathrm{CO}$ emission peak coincident in velocity and position with the cluster. Within our scenario, we speculate that the low-velocity component traces gas that was near to the SSC when it formed but not part of its parent cloud. It may also include clumps that have migrated from the SGMC environment to the cluster neighborhood. This gas would have been too far from the SSC to be accelerated by radiation pressure. This possibility is exemplified by the 30 Doradus starforming region. The gas surrounding the central massive cluster R136 is distributed in an irregular shell, where some molecular clouds that did not participate in the formation of the R136 cluster are observed very close to it ( $\lesssim 10$ pc, Rubio et al. 2009).

We expect the low-velocity molecular gas to be dispersed by the action of stellar winds and future supernova explosions. Stellar winds from massive clusters can erode the surrounding gas and push away the clump material (Rogers \& Pittard 2013).

In the scenario that the gas traced by the low-velocity component is indeed the parent molecular cloud, we do not really understand how the cluster will expel and disrupt its surrounding matter. This component is still very massive, comparable with the mass of the cluster, and, furthermore, it is self-bound. We need observations at higher resolution to resolve this component.

\subsection{Star-formation efficiency}

We are interested to know if SSC B1 is a bound cluster that will evolve as a globular cluster. As described in the introduction, a SFE lower limit of $30 \%$ is needed to create a bound stellar cluster. Within our scenario, for a region of $\sim 100 \mathrm{pc}$ surrounding SSC B1, we estimate a lower limit for the SFE of $17 \%$. In the extreme, unlikely case that both the low- and high-CO velocity components were part of the parent molecular cloud, we estimate $S F E>10 \%$. If we use the Galactic CO conversion factor, then the SFE decreases to $\sim 5 \%$. The estimated value is higher than what is measured in Galactic GMCs, but not high enough to ensure the formation of a bound star cluster. To reach the theoretical SFE limit, at least $30 \%$ of the high-velocity component should not be natal gas but, for example, surrounding gas that was swept-away by stellar feedback. This is possible for SSC B1, which is located in a complex interacting region rich in molecular gas. However, as discussed in the introduction, hydrodynamical simulations indicate that even if the global SFE is low, locally, the SFE can reach values large enough to form a bound cluster (Kruijssen et al. 2012).

Further observations are needed to quantify and test our proposed scenario. Line observations at high resolution of the stellar emission of the cluster would help settle the issues related to the cluster velocity discussed in Sect. 7.1. Higher-resolution observations will reveal the spatial distribution of the gas very close to the cluster, the low-velocity component. With the existing data, we cannot resolve structures smaller than $50 \mathrm{pc}$ and, therefore, we cannot resolve and characterize the clumpy structure of this source. More sensitive observations will enable us to better trace the morphology of the high-velocity component. Also, by looking for high-velocity wings, we can elucidate whether or not the molecular gas is being carried out in a wind together with the hot gas. This can now be done with ALMA, which offers the required angular resolution and sensitivity to address these questions.

\section{Conclusions}

In this paper, we investigate the early evolution of SSC B1 located in SGMC 4/5 in the Antennae overlap region, and, specifically, the impact of the stellar feedback mechanisms on the surrounding matter.

We search for associations between SSCs and compact H II and $\mathrm{H}_{2}$ line emission in four FOVs observed with SINFONI/VLT across the Antennae overlap region. We focus on isolated SSCs previously studied by Gilbert \& Graham (2007). Among our sample, there is only one SSC with both $\mathrm{HII}$ and $\mathrm{H}_{2}$ emission; SSC B1 in SGMC 4/5. This is the only SSC younger than 5 Myr. This is probably not a complete sample since it is difficult to isolate the molecular and ionized emission associated with SSCs. Nevertheless, our sample shows that the association between SSCs and their parent molecular clouds does not last much more than $5 \mathrm{Myr}$; similar to what is found in a sample of SSCs in nearby galaxies and other regions of the Antennae pair (Bastian et al. 2014).

$\mathrm{CO}$ emission in SSC B1 presents two velocity components, a compact, low-velocity one at the position of the SSC, and an 
extended, high-velocity one distributed in a bubble-like shape structure around the cluster. The virial and millimetric masses of the gas associated with SSC B1 low-velocity component are comparable. The comparison with its $\mathrm{CO}$ luminosity yields a CO-to- $\mathrm{H}_{2}$ factor consistent with that found at larger scales $(1.5 \mathrm{kpc})$ and in other starburst galaxies. The detection of molecular gas associated with SSC B1 suggests that the cluster may still be embedded in its parent molecular cloud. Although the actual velocity of the cluster is still unknown and thus it is not clear if the cluster is embedded in this cloud, this source is the only one in the overlap region that could tell us how the molecular gas is being pushed away from the cluster.

We analyzed the physical properties of SSC B1 and the molecular and ionized gas surrounding it. A model of the physical environment of the $\mathrm{H}$ II region and the PDR surrounding SSC $\mathrm{B} 1$ shows that the observed $\mathrm{H}_{2} 2-1 / 1-0 \mathrm{~S}(1)$ intensity line ratio is accounted for by a gas pressure of $3 \times 10^{7}-10^{8} \mathrm{~K} \mathrm{~cm}^{-3}$, which agrees with the estimated radiation pressure from the cluster. This finding implies that there is no significant trapping of the IR photons within the cavity of the molecular gas surrounding the cluster. X-ray observations show that the plasma produced by shocks driven by stellar winds is not confined within a closed shell of cold gas. The plasma pressure is smaller than the radiation pressure. However, radiation pressure is weaker than the gravitational force from the cluster and the cloud itself. It is not strong enough to push away the observed molecular gas.

We propose that radiation pressure was early enhanced and was the main mechanism driving the disruption of the parent molecular cloud in $\sim 1$ Myr. The high-velocity component would be tracing outflowing molecular gas from this disruption. The association of this component with shock heated $\mathrm{H}_{2}$ gas, and the presence of escaping high $\mathrm{Br} \gamma$ gas, are supporting evidence. The low-velocity component may be gas that was near to the SSC when it formed but not part of its parent cloud or clumps that migrated from the SGMC environment. This component would be disrupted by the action of stellar winds and supernova explosions. The existing data do not allow us to conclude whether or not SSC B1 is gravitationally bound and will evolve as a globular cluster. The lower limit for the SFE of $17 \%$, estimated in a region of $100 \mathrm{pc}$ size from the cluster, is slightly smaller than the theoretical limit for bound cluster formation.

Further observations are needed to quantify and test our proposed scenario. Additional observations of line emission from the cluster would allow us to directly measure the velocity of the cluster. Higher spatial and spectral-resolution observations are needed to probe the clumpy structure of the closest component to the cluster.

Acknowledgements. We would like to thank the anonymous referee for the detailed and constructive report that helped us to improve our manuscript. The Atacama Large Millimeter/submillimeter Array (ALMA), an international astronomy facility, is a partnership of Europe, North America and East Asia in cooperation with the Republic of Chile. This paper makes use of the following ALMA Science Verification data: ADS/JAO.ALMA\#2011.0.00003.SV and Early Science data: ADS/JAO.ALMA\#2011.0.00876.S.

\section{References}

Adamo, A., \& Bastian, N. 2015, ArXiv e-prints [arXiv: 1511.08212] Andersen, M., Zinnecker, H., Moneti, A., et al. 2009, ApJ, 707, 1347 Ashman, K. M., \& Zepf, S. E. 2001, AJ, 122, 1888

Bastian, N., Trancho, G., Konstantopoulos, I. S., \& Miller, B. W. 2009, ApJ, 701, 607

Bastian, N., Hollyhead, K., \& Cabrera-Ziri, I. 2014, MNRAS, 445, 378
Beck, S. C., Turner, J. L., Langland-Shula, L. E., et al. 2002, AJ, 124, 2516 Bolatto, A. D., Wolfire, M., \& Leroy, A. K. 2013, ARA\&A, 51, 207 Brogan, C., Johnson, K., \& Darling, J. 2010, ApJ, 716, L51 Castor, J., McCray, R., \& Weaver, R. 1975, ApJ, 200, L107

Clark, J. S., Negueruela, I., Crowther, P. A., \& Goodwin, S. P. 2005, A\&A, 434, 949

Compiegne, M., Verstraete, L., Jones, A., et al. 2011, A\&A, 525, A103

Dale, J. E., \& Bonnell, I. 2011, MNRAS, 414, 321

Dale, J. E., Ercolano, B., \& Bonnell, I. A. 2012, MNRAS, 424, 377

Desert, F.-X., Boulanger, F., \& Puget, J. L. 1990, A\&A, 237, 215

Draine, B. T. 2011, ApJ, 732, 100

Dunne, L., Gomez, H. L., da Cunha, E., et al. 2011, MNRAS, 417, 1510

Elmegreen, B. G., \& Efremov, Y. N. 1997, ApJ, 480, 235

Espada, D., Komugi, S., Muller, E., et al. 2012, ApJ, 760, L25

Fall, S. M., Krumholz, M. R., \& Matzner, C. D. 2010, ApJ, 710, L142

Gilbert, A. M., \& Graham, J. R. 2007, ApJ, 668, 168

Gilbert, A. M., Graham, J. R., McLean, I. S., et al. 2000, ApJ, 533, L57

Guillard, P., Boulanger, F., Pineau des Forêts, G., \& Appleton, P. N. 2009, A\&A, 502,515

Habart, E., Abergel, A., Boulanger, F., et al. 2011, A\&A, 527, A122

Harper-Clark, E., \& Murray, N. 2009, ApJ, 693, 1696

Herrera, C. N., Boulanger, F., \& Nesvadba, N. P. H. 2011, A\&A, 534, A138

Herrera, C. N., Boulanger, F., Nesvadba, N. P. H., \& Falgarone, E. 2012, A\&A, 538, L9

Herrera, C. N., Rubio, M., Bolatto, A. D., et al. 2013, A\&A, 554, A91

Hollyhead, K., Bastian, N., Adamo, A., et al. 2015, MNRAS, 449, 1106

Hummer, D. G., \& Storey, P. J. 1987, MNRAS, 224, 801

Kamenetzky, J., Rangwala, N., Glenn, J., Maloney, P. R., \& Conley, A. 2014, ApJ, 795, 174

Kassis, M., Adams, J. D., Campbell, M. F., et al. 2006, ApJ, 637, 823

Kristensen, L. E. 2007, Ph.D. Thesis, LERMA, Observatoire de Paris-Meudon, LAMAP, Université de Cergy-Pontoise

Kruijssen, J. M. D., Maschberger, T., Moeckel, N., et al. 2012, MNRAS, 419, 841

Krumholz, M. R., \& Matzner, C. D. 2009, ApJ, 703, 1352

Krumholz, M. R., Bate, M. R., Arce, H. G., et al. 2014, Protostars and Planets VI, 243

Le Bourlot, J., Le Petit, F., Pinto, C., Roueff, E., \& Roy, F. 2012, A\&A, 541, A76 Lopez, L. A., Krumholz, M. R., Bolatto, A. D., Prochaska, J. X., \& RamirezRuiz, E. 2011, ApJ, 731, 91

MacLaren, I., Richardson, K. M., \& Wolfendale, A. W. 1988, ApJ, 333, 821

Mathis, J. S., Mezger, P. G., \& Panagia, N. 1983, A\&A, 128, 212

Matzner, C. D. 2002, ApJ, 566, 302

Matzner, C. D., \& McKee, C. F. 2000, ApJ, 545, 364

Mengel, S., Lehnert, M. D., Thatte, N., \& Genzel, R. 2005, A\&A, 443, 41

Mirabel, I. F., Vigroux, L., Charmandaris, V., et al. 1998, A\&A, 333, L1

Murray, N., Quataert, E., \& Thompson, T. A. 2010, ApJ, 709, 191

Neff, S. G., \& Ulvestad, J. S. 2000, AJ, 120, 670

Östlin, G., Cumming, R. J., \& Bergvall, N. 2007, A\&A, 461, 471

Planck Collaboration XXV. 2011, A\&A, 536, A25

Portegies Zwart, S. F., McMillan, S. L. W., \& Gieles, M. 2010, ARA\&A, 48, 431

Rogers, H., \& Pittard, J. M. 2013, MNRAS, 431, 1337

Roman-Lopes, A., \& Abraham, Z. 2004, AJ, 127, 2817

Rubio, M., Paron, S., \& Dubner, G. 2009, A\&A, 505, 177

Ryter, C. E. 1996, Ap\&SS, 236, 285

Sakamoto, K., Ho, P. T. P., Iono, D., et al. 2006, ApJ, 636, 685

Schulz, A., Henkel, C., Muders, D., et al. 2007, A\&A, 466, 467

Schweizer, F., Burns, C. R., Madore, B. F., et al. 2008, AJ, 136, 1482

Scoville, N., Aussel, H., Sheth, K., et al. 2014, ApJ, 783, 84

Skinner, M. A., \& Ostriker, E. C. 2015, ApJ, 809, 187

Smith, L. J., Crowther, P. A., Calzetti, D., \& Sidoli, F. 2016, ApJ, 823, 38 Solomon, P. M., Rivolo, A. R., Barrett, J., \& Yahil, A. 1987, ApJ, 319, 730

Turner, J. L., Ho, P. T. P., \& Beck, S. C. 1998, AJ, 116, 1212

Turner, J. L., Beck, S. C., Benford, D. J., et al. 2015, Nature, 519, 331

Ueda, J., Iono, D., Petitpas, G., et al. 2012, ApJ, 745, 65

Weidner, C., Bonnell, I. A., \& Zinnecker, H. 2010, ApJ, 724, 1503

Weiß, A., Walter, F., Neininger, N., \& Klein, U. 1999, A\&A, 345, L23

Whitmore, B. C., \& Schweizer, F. 1995, AJ, 109, 960

Whitmore, B. C., \& Zhang, Q. 2002, AJ, 124, 1418

Whitmore, B. C., Chandar, R., Schweizer, F., et al. 2010, AJ, 140, 75

Whitmore, B. C., Brogan, C., Chandar, R., et al. 2014, ApJ, 795, 156

Wilson, C., Scoville, N., Madden, S., \& Charmandaris, V. 2000, ApJ, 542, 120

Wilson, C. D., Scoville, N., Madden, S. C., \& Charmandaris, V. 2003, ApJ, 599, 1049

Zezas, A., Fabbiano, G., Baldi, A., et al. 2006, ApJS, 166, 211

Zhu, M., Seaquist, E. R., \& Kuno, N. 2003, ApJ, 588, 243 
Table A.1. Radiation fields and gas pressures exerted at the surface of the PDR, for different values of the internal cavity of the H II region $R_{\text {in }}$.

\begin{tabular}{ccc}
\hline \hline $\begin{array}{c}R_{\text {in }} \\
\mathrm{pc}\end{array}$ & $\begin{array}{c}\chi \\
\text { Mathis }\end{array}$ & $\begin{array}{c}P_{\mathrm{rad}} / k_{\mathrm{B}} \\
\mathrm{K} \mathrm{cm}^{-3}\end{array}$ \\
\hline 30 & $1.6 \times 10^{4}$ & $1.5 \times 10^{8}$ \\
40 & $1.1 \times 10^{4}$ & $8.6 \times 10^{7}$ \\
50 & $7.5 \times 10^{3}$ & $5.5 \times 10^{7}$ \\
60 & $5.5 \times 10^{3}$ & $3.8 \times 10^{7}$ \\
70 & $4.2 \times 10^{3}$ & $2.8 \times 10^{7}$ \\
80 & $3.3 \times 10^{3}$ & $2.1 \times 10^{7}$ \\
90 & $2.7 \times 10^{3}$ & $1.7 \times 10^{7}$ \\
100 & $2.2 \times 10^{3}$ & $1.4 \times 10^{7}$ \\
\hline
\end{tabular}

\section{Appendix A: Modeling the physical environment of SSC B1}

In this section, we introduce a simple geometrical model that we use to quantify the action of the stellar feedback in the parent cloud of SSC B1. The geometrical model is combined with a PDR model to quantify the $\mathrm{H}_{2}$ line emission. We introduce the model in two parts, we model the H II region and then the PDR. This 1D model describes a segment of the shell. It does not assume that the cluster is fully embedded in a spherical shell. The matter around the SSC is likely to be clumpy, with some radiation escaping in some directions. This is in agreement with the fact that the mean extinction of the shell is moderate (a factor 2, Sect. 4.1). The H II region is directly receiving the UV radiation from the SSC and the incident radiation on the PDR is the output radiation from the H II gas layer. The internal radius of the H II cavity $R_{\text {in }}$ is a parameter of the model and the external radius is the GMC radius observed in the continuum emission, $R_{\text {out }}=52 \mathrm{pc}$. The stellar radiation was chosen to be that from the Starburst 99 models, for an age of 3 Myr scaled by the cluster mass. This SED was used for the H II region modeling as the ionizing radiation.

\section{A.1. HIl region}

We model the HII region as an ionized hydrogen nebula with dust grains. We compute the radiative transfer equation for this nebula in the plane-parallel approximation. In this nebula, the radiative transfer will be defined by the absorption produced by the ionization of the hydrogen atoms and light absorption and scattering by dust grains,

$$
\frac{\mathrm{d} I(\lambda)}{\mathrm{d} s}=-n_{\mathrm{H}} \sigma_{\mathrm{H}}(\lambda) I(\lambda)-n_{\mathrm{d}} \sigma_{\mathrm{d}}(\lambda) I(\lambda),
$$

where $s$ is the position in the nebula from $R_{\text {in }}$ at which the absorption occurs, $n_{\mathrm{H}}$ and $n_{\mathrm{d}}$, and $\sigma_{\mathrm{H}}$ and $\sigma_{\mathrm{d}}$ are the hydrogen and dust densities and cross sections, respectively. $\sigma_{\mathrm{H}}$ is zero for non-ionizing photons. We assume that the nebula extent is small compared to $R_{\text {in }}$. The ionization rate $\left(N_{\text {ion }}=n_{\mathrm{H}} \bar{\sigma} S\right)$ equals the recombination rate $\left(N_{\text {rec }}=n_{\mathrm{e}} n_{+} \alpha_{2}\right)$, where $\bar{\sigma}$ is the mean photoionization cross section, $S=S_{0} \mathrm{e}^{-\tau}$ is the number of ionizing photons per unit time and unit area, $n_{\mathrm{e}}$ is the electron density, $n_{+}$

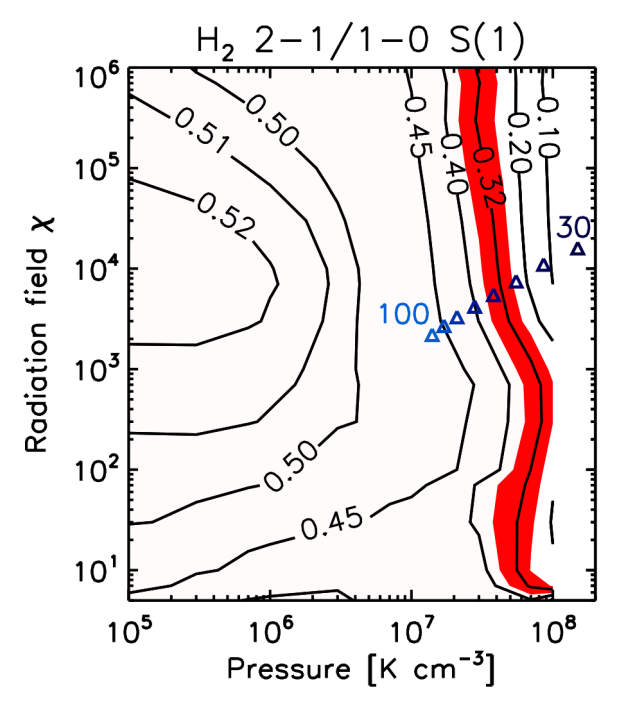

Fig. A.1. $\mathrm{H}_{2}$ 2-1/1-0 S(1) intensity line ratio estimated from the PDR Meudon code for a grid of radiation fields and gas pressures. The measured value for the low-velocity component is 0.32 , which is highlighted in the figure. The red area marks $1 \sigma$ uncertainty associated with this value. Triangles represent the values given in Table A.1, estimated from the $\mathrm{H}$ II/PDR model for radii from 30 to $100 \mathrm{pc}$.

is the density of protons and $\alpha_{2}$ is the recombination coefficient. Then, we can re-write Eq. (A.1) as:

$\frac{\mathrm{de}^{-\tau_{\lambda}}}{\mathrm{d} s}=-\frac{\alpha_{2}}{S_{0}} n^{2}-n x_{\mathrm{d}} \sigma_{\mathrm{d}}(\lambda) \mathrm{e}^{-\tau_{\lambda}}$,

where $n$ is the gas density and $x_{\mathrm{d}}$ is the dust-to-gas mass ratio. We assume that all the protons and electrons come from the same atoms $\left(n=n_{\mathrm{e}}=n_{+}\right)$. The dust cross-sections are taken from the DUSTEM model (Desert et al. 1990; Compiegne et al. 2011). We include the extinction cross sections of the very small grains and big grains (there is no observational evidence for PAHs in H II regions, i.e., Kassis et al. 2006).

We solve the radiative transfer equation (Eq. (A.2)) for different values of incident radiation on the $\mathrm{H}$ II region, depending on the value of the unknown quantity $R_{\text {in }}$, which we vary from 30 to 100 by spacings of $10 \mathrm{pc}$. This range is distributed around the GMC radius ( $\sim 50 \mathrm{pc})$, from the radius of the $\mathrm{Br} \gamma$ line emission to that of $\mathrm{H}_{2}$. We compute the output flux in units of the Mathis radiation field (Mathis et al. 1983), integrating the emission between 91.2 to $200 \mathrm{~nm}$. Table A.1 lists the radiation field for the different internal radii of the cavity. We also compute the radiation pressure exerted at the surface of the PDR from photons absorbed or scattered across the layer of the H II gas,

$P_{\mathrm{rad}}(\mathrm{HII})=\frac{L_{\mathrm{HII}}}{4 \pi R_{\mathrm{in}}^{2} c}$,

where the luminosity $L_{\mathrm{HII}}$ is the luminosity incident onto the H II region minus the output luminosity. $P_{\text {rad }}(\mathrm{HII})$ is the pressure produced by the ionizing photons and the non-ionizing photons, which are absorbed by dust and gas in the HII layer. This is the minimum value of the pressure at the surface of the PDR. Table A.1 lists the estimated values of $P_{\text {rad }}$. 


\section{A.2. PDR}

To describe the PDR, we use the online tables of outputs from the PDR Meudon Code $^{2}$ (Le Bourlot et al. 2012). We chose to use results from isobaric rather than isochoric models to relate line intensities to the radiation pressure. Model results are available for a range of values of the incident radiation field and gas pressures. The model grid ranges are $P / k_{\mathrm{B}}=n T_{\mathrm{K}}=$ $1 \times 10^{5}-1 \times 10^{8} \mathrm{~K} \mathrm{~cm}^{-3}$, for the gas pressure and $\chi=1$ to $1 \times 10^{6}$ for the radiation field in Mathis units.
Figure A.1 shows the model results for the $\mathrm{H}_{2} 2-1 / 1-0 \mathrm{~S}(1)$ line ratio. The red filled area indicates the $1 \sigma$ uncertainty on the observed ratio for the low-velocity component (Table 4). The range of possible combinations of radiation fields and pressures given in Table A.1 are marked by triangles. This figure indicates that PDR models can account for the observed intensity ratios within the constrains on physical parameters set by the spatial extent of the emission lines. In the paper, we use a value $R_{\text {in }}=$ $35 \mathrm{pc}$, which corresponds to the value observed in $\mathrm{Br} \gamma$. From the model in Fig. A.1, the best $R_{\text {in }}$ value is $\sim 55$ pc. 